\title{
Identification and characterization of putative xylose and cellobiose transporters in Aspergillus nidulans
}

Thaila Fernanda dos Reis ${ }^{1}$, Pollyne Borborema Almeida de Lima², Nádia Skorupa Parachin², Fabiana Bombonato Mingossi ${ }^{3}$, Juliana Velasco de Castro Oliveira ${ }^{3}$, Laure Nicolas Annick Ries ${ }^{1}$ and Gustavo Henrique Goldman ${ }^{1^{*}}$

\begin{abstract}
Background: The conversion of lignocellulosic biomass to biofuels (second-generation biofuel production) is an environmentally friendlier alternative to petroleum-based energy sources. Enzymatic deconstruction of lignocellulose, catalyzed by filamentous fungi such as Aspergillus nidulans, releases a mixture of mono- and polysaccharides, including hexose (glucose) and pentose (xylose) sugars, cellodextrins (cellobiose), and xylooligosaccharides (xylobiose). These sugars can subsequently be fermented by yeast cells to ethanol. One of the major drawbacks in this process lies in the inability of yeast, such as Saccharomyces cerevisiae, to successfully internalize sugars other than glucose. The aim of this study was, therefore, to screen the genome of $A$. nidulans, which encodes a multitude of sugar transporters, for transporters able to internalize non-glucose sugars and characterize them when introduced into $S$. cerevisiae.

Results: This work identified two proteins in A. nidulans, CltA and CltB, with roles in cellobiose transport and cellulose signaling, respectively. CltA, when introduced into $\mathrm{S}$. cerevisiae, conferred growth on low and high concentrations of cellobiose. Deletion of $c l t B$ resulted in reduced growth and extracellular cellulase activity in $A$. nidulans in the presence of cellobiose. CltB, when introduced into $S$. cerevisiae, was not able to confer growth on cellobiose, suggesting that this protein is a sensor rather than a transporter. However, we have shown that the introduction of additional functional copies of $\mathrm{CltB}$ increases the growth in the presence of low concentrations of cellobiose, strongly indicating CltB is able to transport cellobiose. Furthermore, a previously identified glucose transporter, $\mathrm{HxtB}$, was also found to be a major xylose transporter in A. nidulans. In S. cerevisiae, HxtB conferred growth on xylose which was accompanied by ethanol production.

Conclusions: This work identified a cellobiose transporter, a xylose transporter, and a putative cellulose transceptor in A. nidulans. This is the first time that a sensor role for a protein in A. nidulans has been proposed. Both transporters are also able to transport glucose, highlighting the preference of $A$. nidulans for this carbon source. This work provides a basis for future studies which aim at characterizing and/or genetically engineering Aspergillus spp. transporters, which, in addition to glucose, can also internalize other carbon sources, to improve transport and fermentation of non-glucose sugars in S. cerevisiae.
\end{abstract}

Keywords: Aspergillus nidulans, Saccharomyces cerevisiae, Xylose, Cellobiose, Sugar transport

\footnotetext{
*Correspondence: ggoldman@usp.br

1 Departamento de Ciências Farmacêuticas, Faculdade de Ciências

Farmacêuticas de Ribeirão Preto, Universidade de São Paulo, Av. do Café

S/N, Ribeirão Preto, SP CEP 14040-903, Brazil

Full list of author information is available at the end of the article
} 


\section{Background}

An increase in energy demands, a depletion in fossil fuels, and high emissions of greenhouse gases have led to the search for alternative and environmentally friendlier energy sources. One alternative energy source is lignocellulose which is found in the cell walls of all plants, such as hardwoods, softwoods, crops, and grasses, thus making it the most abundant organic material on the planet [1-4]. Lignocellulosic wastes are produced by the forestry, pulp and paper, and agriculture industries in addition to municipal and animal wastes [5]. The main components of lignocellulosic biomass are cellulose (40-50\%), hemicelluloses (25-35\%) and lignin (15-20\%) [6]. Cellulose consists of long chains of the hexose sugar glucose, which represents the most abundant simple sugar in the plant cell wall, whereas the main sugar of hemicelluloses is xylose, although other sugars, such as arabinose and galactose, also make up considerable fractions of this polysaccharide. The production of biofuels from lignocellulose, in a process called 2nd generation (2G) biofuel production, aims at converting these sugars into ethanol [7]. Lignocellulosic biomass is deconstructed by enzymatic degradation into a mixture of hexose (e.g., glucose) sugars, pentose (e.g., xylose) sugars, cellodextrins (e.g., cellobiose), and xylooligosaccharides. Cellodextrins are glucose polymers of varying lengths (e.g., cellobiose is a glucose dimer), released during cellulose degradation by cellobiohydrolases and which are subsequently cleaved into glucose monomers by $\beta$-glucosidases $[5,8,9]$. Once these simpler sugars have been released from the complex lignocellulosic polymers by enzymatic deconstruction, they can be converted into ethanol by fermenting organisms.

The preferred organism for fermentation of lignocellulosic sugars to ethanol is the budding yeast Saccharomyces cerevisiae, which is substantially used in several industrial processes, such as baking, brewing, and wine making [10]. S. cerevisiae primarily uses glucose monomers for fermentation and is unable to ferment cellobiose. Furthermore, S. cerevisiae is also unable to grow efficiently on xylose as the sole carbon source, although its genome appears to encode all components necessary for metabolizing xylose [11]. Genetic engineering of $S$. cerevisiae has introduced components into the yeast cells that allowed fermentation of cellobiose and xylose $[12,13]$, but transport of these sugars into the cell is still a limiting factor for successful conversion to ethanol. Complete fermentation of all the sugars found in lignocellulose is desired to reduce the costs of $2 \mathrm{G}$ biofuel production and make it an economically feasible process [14]. Therefore, one of the bottlenecks of the conversion of lignocellulose to ethanol lies in the engineering of yeast strains, which can efficiently transport xylose, cellobiose and other lignocellulosic sugars into the cell [15].
Transport of carbon sources is mainly carried out by single polypeptide secondary carriers belonging to the major facilitator superfamily (MFS) of transporters and which transport small soluble molecules in response to ion gradients $[16,17]$. The MFS of transporters is divided into 17 families of which families 1,5 , and 7 are involved in sugar transport $[16,17]$. Domestic and wild-type $S$. cerevisiae species transport xylose into the cell with low affinity $\left(K_{M}=100-190 \mathrm{mM}\right)$ via the expression of native high-affinity hexose transporter-encoding genes, such as GAL2 and HXT7 [18, 19], highlighting the preference of $S$. cerevisiae for glucose. Although specific pentose transporters have not been described in yeast, engineering of hexose transporters has been shown to significantly improve xylose transport [20-22]. Furthermore, heterologous introduction of specific D-xylose transporters, derived from other organisms, can improve the growth rate of S. cerevisiae on xylose, increasing $\mathrm{V}_{\max }$ (maximum reaction velocity rate) values and displaying an increase in high cell density sugar consumption [23]. However, this heterologous system only supports low rates of D-xylose transport $[24,25]$ and may not be perfectly integrated in the endogenous carbon metabolism regulatory network of S. cerevisiae. Similar to xylose transporters, cellodextrin transporters from Neurospora crassa have also been introduced into $S$. cerevisiae together with a $\beta$-glucosidase-encoding gene; they conferred the ability of S. cerevisiae to grow on cellobiose [26]. The advantages of $S$. cerevisiae being able to directly use cellobiose for growth are that this does not require adding large quantities of $\beta$-glucosidases into the cultures and it also prevents the build-up of glucose in the culture medium that is repressive for cellulase and hemicellulase-encoding genes [27]. However, further engineering is required to optimize cellobiose transport and metabolism in S. cerevisiae. The search to find xylose and cellobiose-specific transporters is, therefore, of importance for bioethanol production from lignocellulose.

Filamentous fungi degrade lignocellulosic biomass through secreting a large repertoire of hydrolytic enzymes that break down lignocellulosic sugar polymers into simple sugars which subsequently can be taken up by the cell $[28,29]$. Accordingly, the genomes of filamentous fungi also encode large numbers of MFS transporters. Currently, the genomes of Trichoderma reesei and $A$. nidulans are predicted to encode 164 and 357 proteins, respectively, belonging to the MFS, although it is not known how many of these are exactly involved in sugar transport [30, 31]. In addition, filamentous fungi, such as $N$. crassa and T. reesei, are able to transport disaccharides such as cellobiose into the cell through cellobiosespecific transporters; once internalized, cellobiose has been shown to play an important role in signaling the 
presence of cellulose [12, 19, 32]. Furthermore, transporters expressed by filamentous fungi often can transport more than one type of sugar; for example, the $A$. nidulans transporter XtrD was shown to be able to transport, in addition to xylose and glucose, several other monosaccharides, whereas the T. reesei STP1 transporter is involved in glucose and cellobiose uptake [32, 33]. However, a very few sugar transporters have been functionally characterized in filamentous fungi [34-40].

The aim of this work was, therefore, to identify and characterize $A$. nidulans transporters involved in cellobiose and xylose uptake and heterologously introduce them into S. cerevisiae. This study identified several transporters with roles in pentose or cellodextrin transport. Characterization of the cellobiose transporter CltA showed increased efficiency in cellobiose transport than when compared to a previously identified $N$. crassa cellobiose transporter. Furthermore, this work identified CltB as a putative cellobiose transceptor. In addition, a previously described glucose transporter was identified as playing a major role in xylose transport.

\section{Results \\ Identification of $\mathrm{CltA}$ and $\mathrm{CltB}$ with roles in cellobiose transport or signaling}

We have previously used genome-wide transcriptional profiling to identify 12 transporters, belonging to the major facilitator superfamily (MFS) that have increased mRNA accumulation in xylose-rich conditions [33]. One of these transporters, named $\mathrm{XtrD}$, was identified as a xylose-specific transporter [33]. We, therefore, started by characterizing three other randomly chosen transporters ( $x$ trF- $H$ that correspond to AN0332, AN8347, and AN9173, respectively) that belong to this series of putative xylose transporters. Although these three genes were upregulated in the presence of xylose, deletion of these genes in A. nidulans did not have a significant effect on growth in the presence of xylose and glucose nor could they confer growth, when heterologously introduced, of S. cerevisiae in the presence of xylose, glucose, and other monosaccharides (data not shown). Since these transporters were not able to transport either hexoses or pentoses, we hypothesized if they could be involved in the transport of more complex sugars, such as cellodextrins (e.g., cellobiose) or xylooligosaccharides. The genome of $N$. crassa encodes two cellobiose transporters termed $C D T-1$ and $C D T-2$ which transport and internalize cellodextrin molecules $[26,41]$ and which also appear to have transceptor activity and, therefore, play a role in cellulose signaling [42]. The here identified, supposed xylose transporter-encoding gene $x \operatorname{trG}$ (AN8347) has identity with the $N$. crassa cellobiose transporter CDT-2 (44 \% identity, $61 \%$ similarity, e-value of $4 \mathrm{e}-137)$. BLASTp search of the
Aspergillus genome database (www.aspgd.org) using $N$. crassa $C D T-1$ as a query allowed us to identify a second gene, AN2814, with high identity to the $N$. crassa cellodextrin transporter (61 \% identity, $75 \%$ similarity, e-value of 0.0 ).

To further characterize these potential cellobiose transporter-encoding genes (here now named $\operatorname{clt} A$ and $\operatorname{clt} B$, respectively), we evaluated their expression patterns in the presence of $1 \%$ cellobiose (Fig. 1a, b). The expression of $c l t A$ increased gradually (about 4.8 -fold) over a time period of $4 \mathrm{~h}$, whereas expression of $c l t B$ varied during the same time period (Fig. 1a, b). Next, both genes were deleted in $A$. nidulans and a $\triangle c l t A \Delta c l t B$ double deletion strain was constructed. The wild-type, $\Delta c l t A, \Delta c l t B$, and the double $\triangle$ clt $A \Delta c l t B$ strains were grown in $1 \%$ glucose and $1 \%$ cellobiose for 48 and $72 \mathrm{~h}$, and biomass was determined (Fig. 1c, d). All the mutant strains had a similar biomass than the wild-type strain when grown in $1 \%$ glucose (Fig. 1c). However, in the presence of cellobiose, the $\Delta c l t B$ strain showed a $\sim 50 \%$ reduction in biomass after $48 \mathrm{~h}$ growth when compared to the wild-type strain, whereas there was no significant difference between the $\triangle c l t A$ and wild-type strains (Fig. 1d). The double mutant showed a $\sim 75 \%$ reduction in biomass when compared to the wild-type strain after 48 -h growth in $1 \%$ cellobiose (Fig. 1d). These results suggest that CltA and CltB could collaborate towards cellobiose transport. Interestingly, there is also a reduction in cellulase activity in the $\triangle c l t B$ and $\Delta c l t A \Delta c l t B$ mutants of 50 and $70 \%$, respectively, than when compared to the wild-type strain (Fig. 1e), suggesting that these transporters play a role in the regulation/signaling of cellulase production.

We decided to investigate in more detail the phenotype provided by $\Delta c l t B$ by complementing and overexpressing the $c l t B$. First, we complemented the $\Delta c l t B$ with a wildtype copy of $c l t B$ integrated ectopically, creating a strain $\triangle c l t B:: c l t B^{+}$. Subsequently, we transformed the wild-type GR5 strain with CltB::GFP and selected for transformants with a single homologous integration and multiple ectopic integrations (Additional file 1). We selected single candidates for homologous (named CltB::GFP) and multiple ectopic integrations (named oCltB3::GFP). Growth phenotypes of $\Delta c l t B:: c l t B^{+}$, CltB::GFP, and oCltB3::GFP were identical to the wild-type strain on MM with glucose as single carbon source (data not shown). Expression measured by qRT-PCR experiments showed that oCltB3::GFP has about eightfold more $c l t B$ expression than the wildtype strain in the presence of cellobiose (Fig. 2a). To verify the cellular localization and expression of CltB:GFP, the GFP strain was grown for $16 \mathrm{~h}$ in fructose and transferred to either 0.1 or $1 \%$ cellobiose for 4 or $8 \mathrm{~h}$ (Fig. $2 \mathrm{~b}$ ). We have not observed any fluorescence in fructose (data not shown), but in contrast in $1 \%$ cellobiose, we were able to 

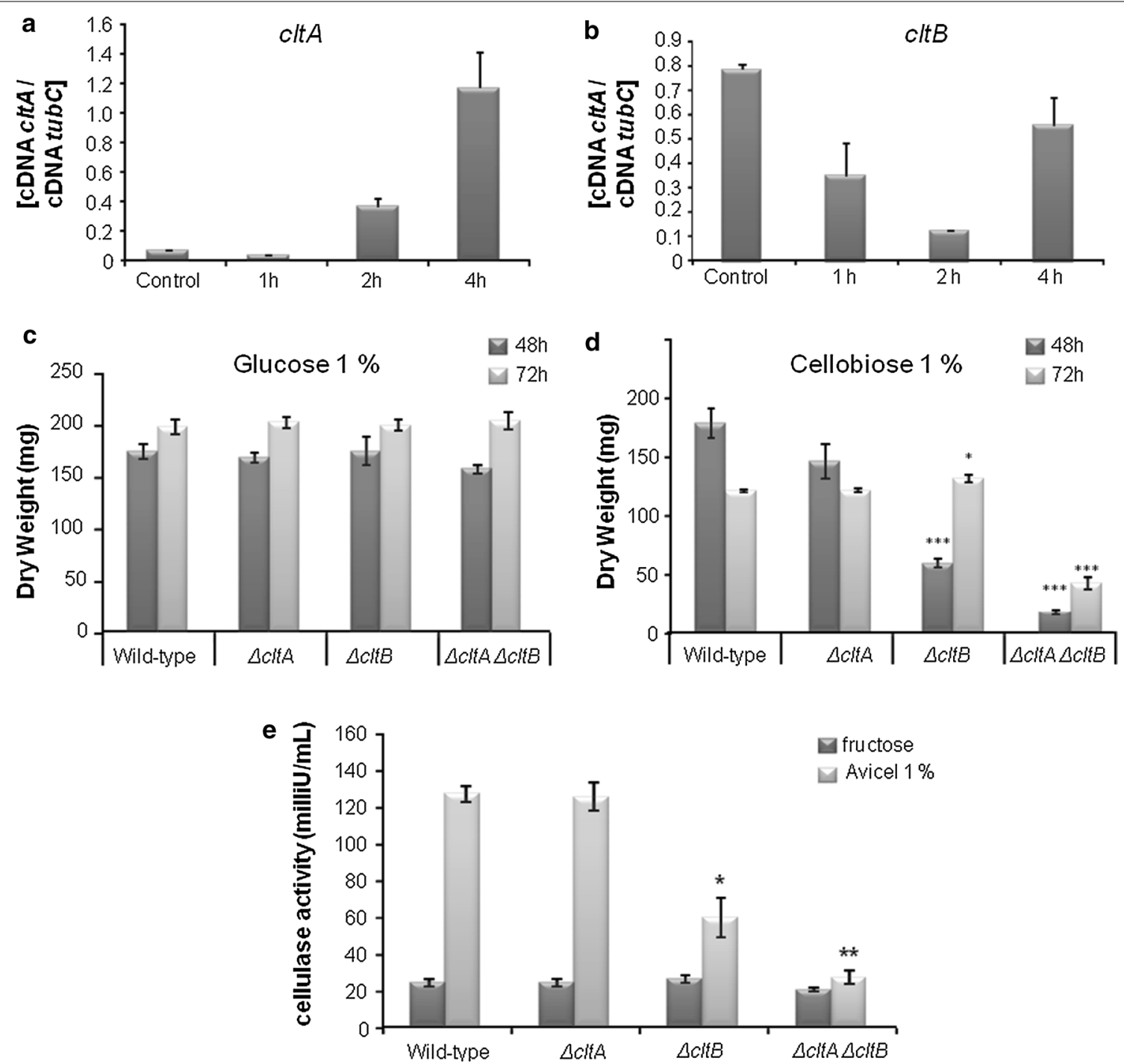

Fig. $1 \mathrm{Clt} A$ and $\mathrm{CltB}$ are cellobiose transporters which are involved in the signaling response to cellulose. The expression of $c l t A(\mathbf{a})$ and $c l t B(\mathbf{b})$ was assessed by RT-qPCR in the presence of $1 \%$ cellobiose. The effect of the single deletions of $c l t A$ and $c l t B$ and the double deletion clt $A$ clt $B$ was assessed on fungal biomass accumulation (DW dry weight) when grown for 48 or $72 \mathrm{~h}$ in the presence of $1 \%$ glucose (c) or $1 \%$ cellobiose (d). Cellulase activity (e) was measure in the supernatants of the same strains when grown in minimal medium supplemented with $1 \%$ fructose for $16 \mathrm{~h}$ and then transferred to minimal medium containing $1 \%$ avicel for 5 days. Error bars indicate standard deviation for three biological replicates

see a weak fluorescence in oCltB3::GFP, mostly localized in the cytoplasm and in the cell membrane (Fig. 2b). To evaluate the impact of overexpressing $c l t B^{+}$on growth in the presence of 0.5 and $1 \%$ cellobiose as a single carbon source, the wild-type, $\Delta c l t B, \quad \Delta c l t B:: c l t B^{+}$, and oCltB3::GFP were grown for $24 \mathrm{~h}$ in $\mathrm{MM}+0.5$ or $1 \%$ cellobiose (Fig. 2c). There is no significant difference in the growth (as evalutated by dry weight) of the wild type and $\Delta c l t B:: c l t B^{+}$in both 0.5 and $1 \%$ cellobiose (Fig. $2 \mathrm{c}$ ); in contrast, as it is also shown in Fig. 1d, we have observed a significant differential reduced growth in $\Delta c l t B$ in both cellobiose concentrations (Fig. 2c). The overexpression strain oCltB3::GFP has shown more growth than the wild type only in $0.5 \%$ but not in $1 \%$ cellobiose (Fig. 2c). Taken together, these results suggest that CltB is able to transport cellobiose.

\section{$\mathrm{CltA}$ and $\mathrm{CltB}$ confer the ability of $\mathrm{S}$. cerevisiae to grow in the presence of cellobiose as sole carbon source}

To evaluate the ability of CltA and CltB to transport cellobiose, both genes were cloned into S. cerevisiae SC9721_ pGH1-1, a SC9721 strain previously transformed with the N. crassa $\beta$-glucosidase-encoding gene gh1-1 (NCU00130) [26]. Both $c l t A$ and $c l t B$ were fused to $g f p$, and plasma 


\section{a}

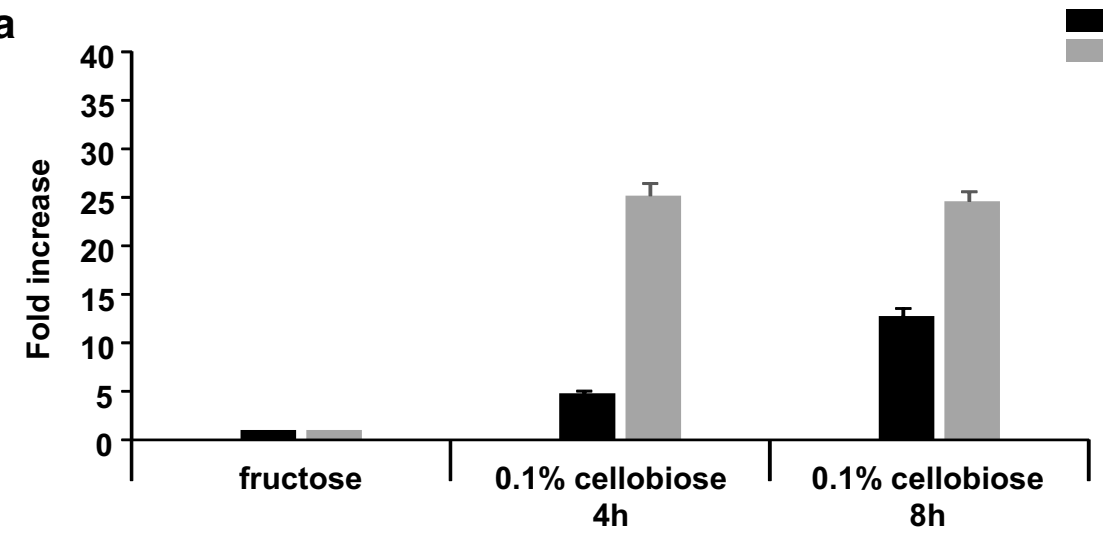

b

$4 \mathrm{~h}$

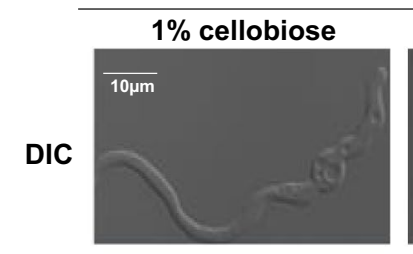

\section{$0.1 \%$ cellobiose}
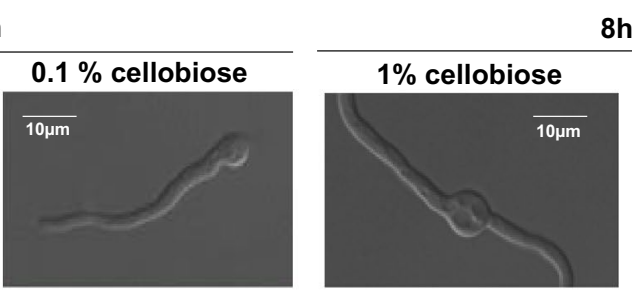

$8 \mathrm{~h}$
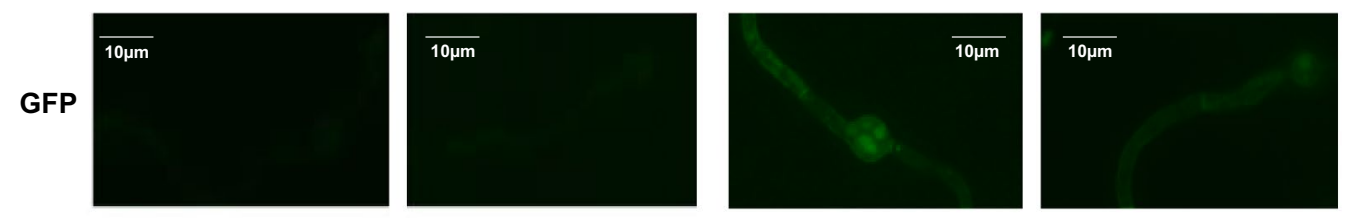

C

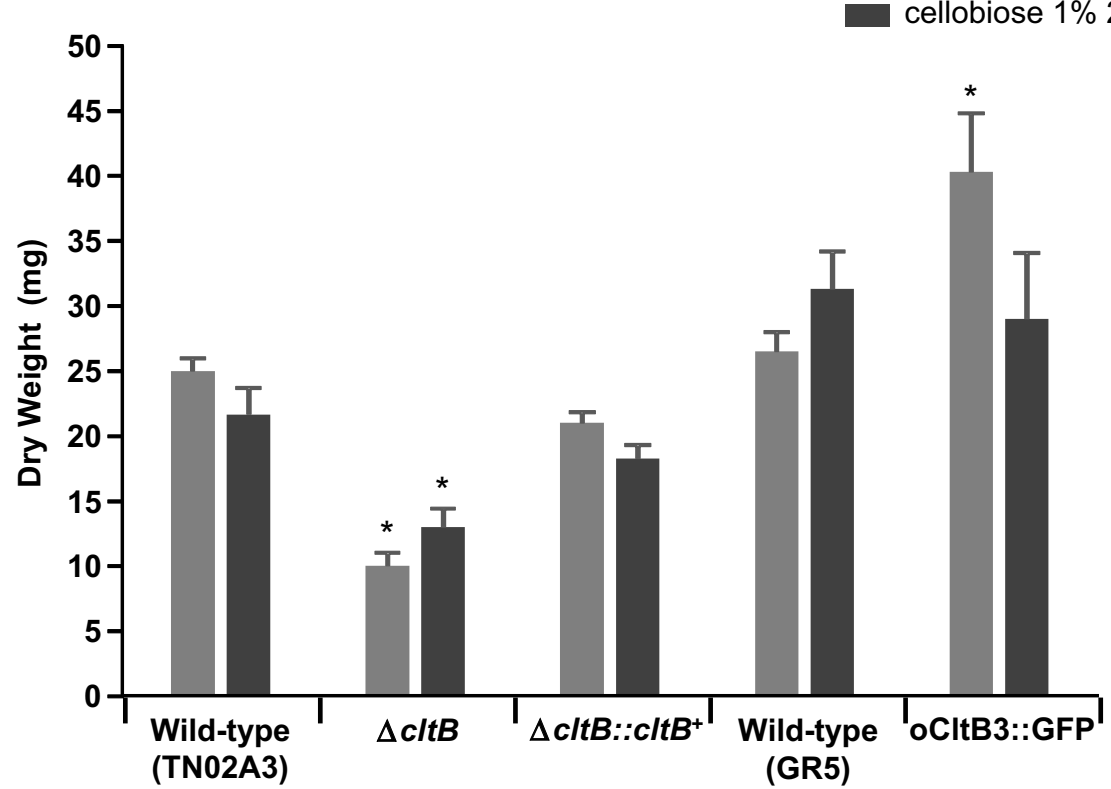

Fig. 2 CltB overexpression increases the growth on cellobiose. a The expression of $c l t B$ was assessed by qRT-PCR in the presence of $0.1 \%$ cellobiose. $\mathbf{b}$ Germlings of oCltB3::GFP were grown for $16 \mathrm{~h}$ in fructose $1 \%$ and transferred to 0.1 or $1 \%$ cellobiose. c The fungal biomass accumulation (dry weight) in the wild-type (TN02A3 and GR5), $\triangle c \mid t B, \triangle C l t B:: c l t B^{+}$, and oCltB3::GFP strains was assessed for $24 \mathrm{~h}$ in the presence of 0.5 or $1 \%$ cellobiose. ${ }^{*} p<0.005$ 
membrane localization of CltA and CltB was confirmed by fluorescence microscopy when grown for $24 \mathrm{~h}$ in YNB supplemented with $1 \%$ glucose medium (Fig. 3a). The S. cerevisiae CltA::GFP and CltB::GFP strains were then grown in liquid YNB medium supplemented with $1 \%$ glucose for 24 $\mathrm{h}$ at $30^{\circ} \mathrm{C}$, before cells were washed and spotted in a serial dilution onto YNB solid medium containing either $1 \%$ glucose or varying concentrations of cellobiose. Yeast strains containing only CltA or CltB (no $\beta$-glucosidase) were used as negative controls as they were unable to grow on cellobiose as sole carbon source. S. cerevisiae transformed with $N$. crassa $c d t$ - 1 and the $\beta$-glucosidase-encoding gene (gh11) was used as a positive control (Fig. 3b). The drop-out assay clearly shows that CltA, and to a lesser extent CltB, is able to transport cellobiose and, thus, enable $S$. cerevisiae to grow on cellobiose as sole carbon source. a

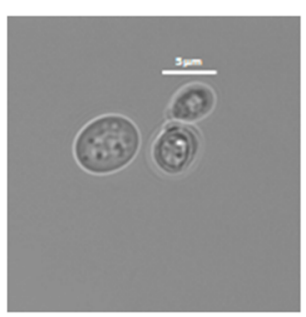

XtrG/CltA::GFP

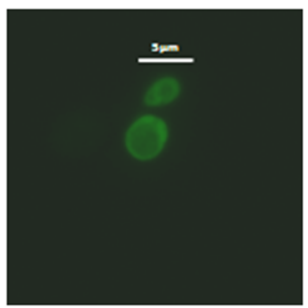

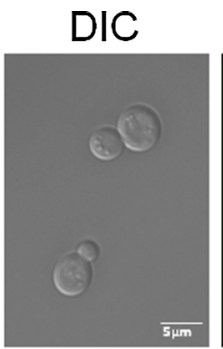

CltB::GFP

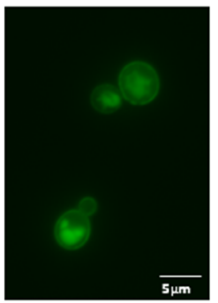

b

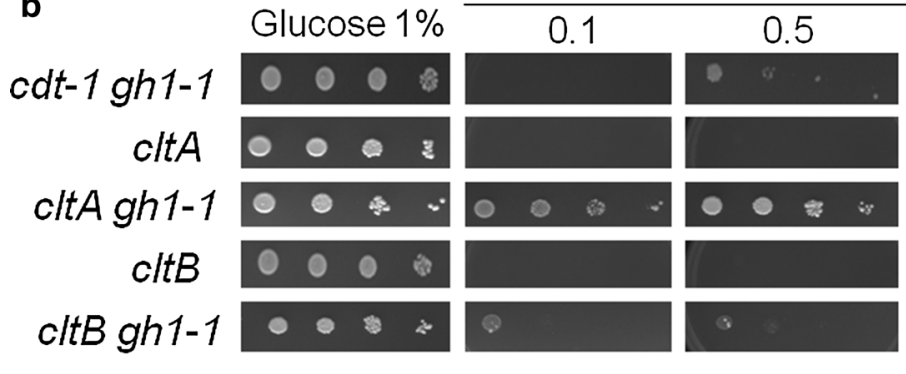

Cellobiose (\%)
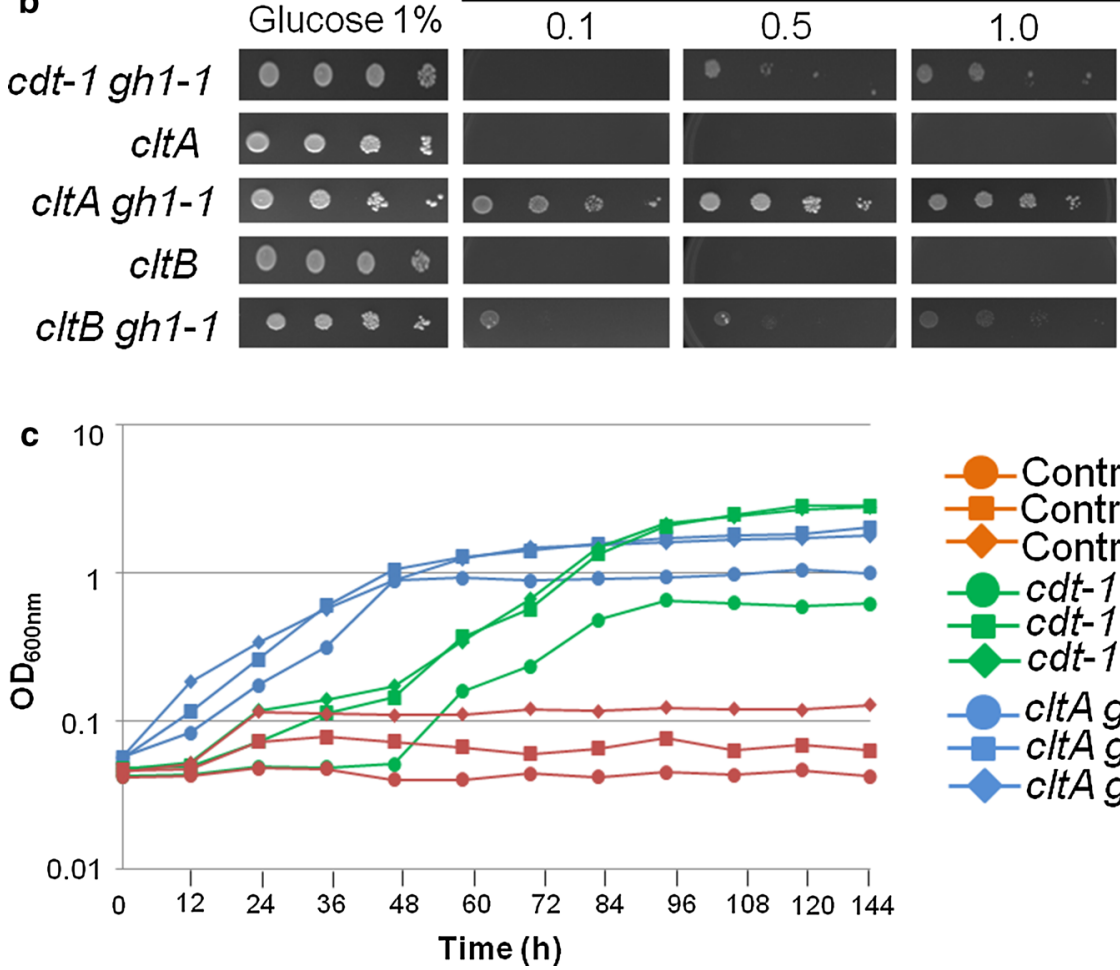

2.0
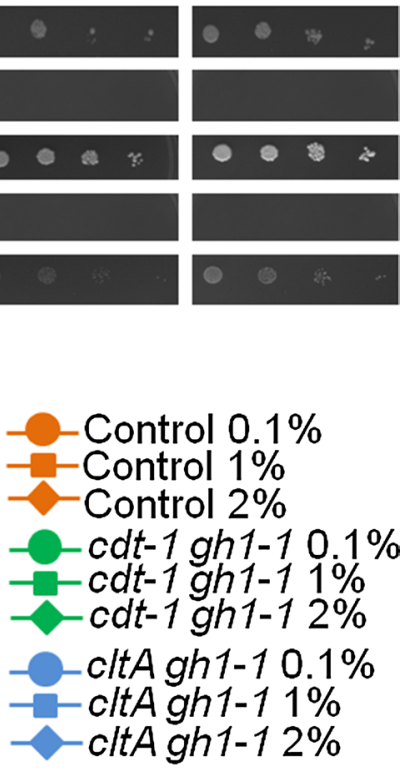

Fig. $3 \mathrm{CltA}$ and CltB confer growth of S. cerevisiae in the presence of low and high concentrations of cellobiose. $\mathbf{a}$ CltA and CltB localize to the plasma membrane in S. cerevisiae. Strains were grown for $16 \mathrm{~h}$ at $30^{\circ} \mathrm{C}$ in YNB supplemented with $2 \%$ glucose before pictures were taken without (DIC) and with (GFP) fluorescence. b S. cerevisiae strains containing A. nidulans CltA, cltB, and N. crassa cdt-1 with and without the $\beta$-glucosidaseencoding (gh 1-1) gene were pre-grown for $24 \mathrm{~h}$ in YNB media containing $1 \%$ glucose before a serial dilution was made (1:10 dilution starting at optical density $\mathrm{OD}_{600 \mathrm{~nm}} 1.0$ ) and diluted cells were grown on YNB plates containing different concentrations of cellobiose $(0.1-2 \%)$. c Growth curves of S. cerevisiae strains containing the N. crassa cdt-1 and the A. nidulans cltA transporter-encoding genes. Both strains also contained the $\beta$-glucosidase-encoding gene gh 1-1. Strains were grown for $144 \mathrm{~h}$ in the presence of different concentrations of cellobiose $(0.1,1$, and $2 \%)$ at $30{ }^{\circ} \mathrm{C}$. Growth was assessed by measuring the OD at $600 \mathrm{~nm}$. The yeast strain expressing CltB has not grown in liquid medium, and it was not used in this experiment 
To compare the efficiency of these cellobiose transporters, the growth rates of $S$. cerevisiae strains harboring either the $A$. nidulans CltA or the $N$. crassa CDT-1 transporters were compared in the presence of different concentrations of cellobiose. Although cltA was expressed from a weaker yeast promoter than $c d t-1$ [43-45], the $S$. cerevisiae CltA strain grew much faster during the first $36 \mathrm{~h}$ incubation in different concentrations of cellobiose, suggesting that CltA appears to transport different concentrations of cellobiose faster into the yeast cell than when compared to the yeast strain containing CDT-1 (Fig. 3c).

\section{Deletion of the hexose transporter-encoding gene hxtB results in reduced xylose uptake}

As mentioned in the introduction, many sugar transporters expressed by filamentous fungi are capable of transporting more than one type of monosaccharide across the fungal membrane. As a next step, we decided to investigate the possibility that $A$. nidulans hexose transporters could be involved in xylose uptake. In a previous study, four hexose transporters, termed HxtB-E were shown to confer growth of $S$. cerevisiae strain EBY. VW4000 in the presence of glucose, fructose, mannose, and galactose [46]. These transporters, therefore, seem to accept multiple sugars as substrates, although xylose as a potential substrate for these transporters was not characterized at that time. In A. niger, on the other hand, MstA, the orthologue of HxtB in A. nidulans was shown to have high affinity for xylose when introduced into $S$. cerevisiae [47]. We, therefore, decided to investigate whether these four transporters were able to transport xylose into the cell.

The $A$. nidulans wild-type strain was first grown from spores in fructose-rich media before being transferred to media containing either 0.2 or $2 \%$ xylose for $6,12,18$ and $24 \mathrm{~h}$ (Fig. 4). Gene expression of $h x t B-E$ was assessed by RT-qPCR in these conditions. All four genes were induced to a different extent in the presence of low concentrations of xylose $(0.2 \%)$ but not in the presence of $2 \%$ xylose (Fig. 4a-d). Next, the four transporter-encoding genes were knocked out in A. nidulans and growth of these strains in the presence of glucose and xylose was assessed. The wild-type and the four deletion strains were grown in liquid minimal medium supplemented with $1 \%$ glucose, $0.2 \%$ xylose or $2 \%$ xylose for 24 and $48 \mathrm{~h}$ before fungal dry weight was measured (Fig. 5). All strains showed a similar biomass when grown in $1 \%$ glucose for 24 and $48 \mathrm{~h}$ (Fig. 5a). The $\Delta h x t B$ and $\Delta h x t E$ strains showed significantly reduced biomass when grown in $2 \%$ xylose for 48 h (Fig. 5b). However, after $72 \mathrm{~h}$ of growth, all the mutant strains had a similar dry weight to the wild-type strain (data not shown). To further characterize xylose uptake, the concentration of xylose was measured in the supernatants of the wild-type, $\Delta h x t B$ and $\Delta h x t E$ strains when grown for $72 \mathrm{~h}$ in medium supplemented with either 1 or $2 \%$ xylose. After $72 \mathrm{~h}$, the wild-type strain and $\Delta h x t E$ strains had consumed all the xylose in the extracellular medium, whereas xylose consumption was much slower in the $\Delta h x t B$ strain and residual xylose could still be detected after $72 \mathrm{~h}$ in the supernatant of this strain (Table 1).

To confirm the above described results, the capacity of xylose uptake was studied in both the wild-type and $\Delta h x t B$ strains using ${ }^{14} \mathrm{C}$-xylose. In the wild-type strain, ${ }^{14} \mathrm{C}$-xylose uptake obeyed single saturation kinetics with a $K_{m}$ value of $56.17 \pm 11.9 \mathrm{mM}$ and a $V_{\max }$ of $0.27 \mu \mathrm{mol}$ of xylose $\mathrm{h}^{-1}$ per $2.5 \times 10^{7}$ conidia (Fig. $5 \mathrm{c}$; Additional file 2 for Eadie-Hofstee and Lineweaver-Burk plots). The $\triangle h x t B$ mutant strain showed both a decreased affinity for xylose $\left(K_{m}=100.4 \pm 17.92 \mathrm{mM}\right)$ and a slight reduction in transport capacity $\left(V_{\max }=0.20 \mu \mathrm{mol}\right.$ of xylose per hour per $2.5 \times 10^{7}$ conidia; Fig. 5c; Additional file 1 for Eadie-Hofstee and Lineweaver-Burk plots).

\section{HxtB confers growth of $S$. cerevisiae in the presence of xylose}

To confirm the presence and the cellular localization of $\mathrm{HxtB}$, the HxtB::GFP and HxtE::GFP strains were constructed. Growth phenotypes of HxtB::GFP and HxtE::GFP were identical to the wild-type strain (data not shown). Both strains were grown for 10, 15, 20, and $24 \mathrm{~h}$ in minimal medium containing 0.1 or $1 \%$ xylose. HxtB::GFP and HxtE::GFP were expressed in the presence of low and high concentrations of xylose upon which it localized to the fungal plasma membrane and small vacuoles (Fig. 6; Additional file 3). To confirm the xylosetransporting capacity of HxtB::GFP, it was introduced into S. cerevisiae EBY.VW4000 strain which was previously transformed with all the components necessary for the xylose metabolic pathway (see "Methods" section). The S. cerevisiae EBY.VW4000 strain lacks around 20 glucose transporters and is unable to grow on various hexose and pentose monosaccharides, including glucose, fructose, mannose, galactose, and xylose [48]. This strain is, therefore, a good tool for evaluating the ability of heterologous introduced transporter to take up various sugars thus conferring growth to $S$. cerevisiae in the presence of various pentose and hexose sugars. HxtB::GFP localized to the plasma membrane in $S$. cerevisiae when grown in maltose-rich conditions (Fig. 7a). Furthermore, when transferred from maltose-rich media to media containing low and high concentrations of xylose, S. cerevisiae strain HxtB::GFP was able to grow in both 0.1 and $1 \%$ xylose, whereas the strain which lacked HxtB::GFP (control) was not able to do it (Fig. 7b). Furthermore, ${ }^{14} \mathrm{C}$-xylose 

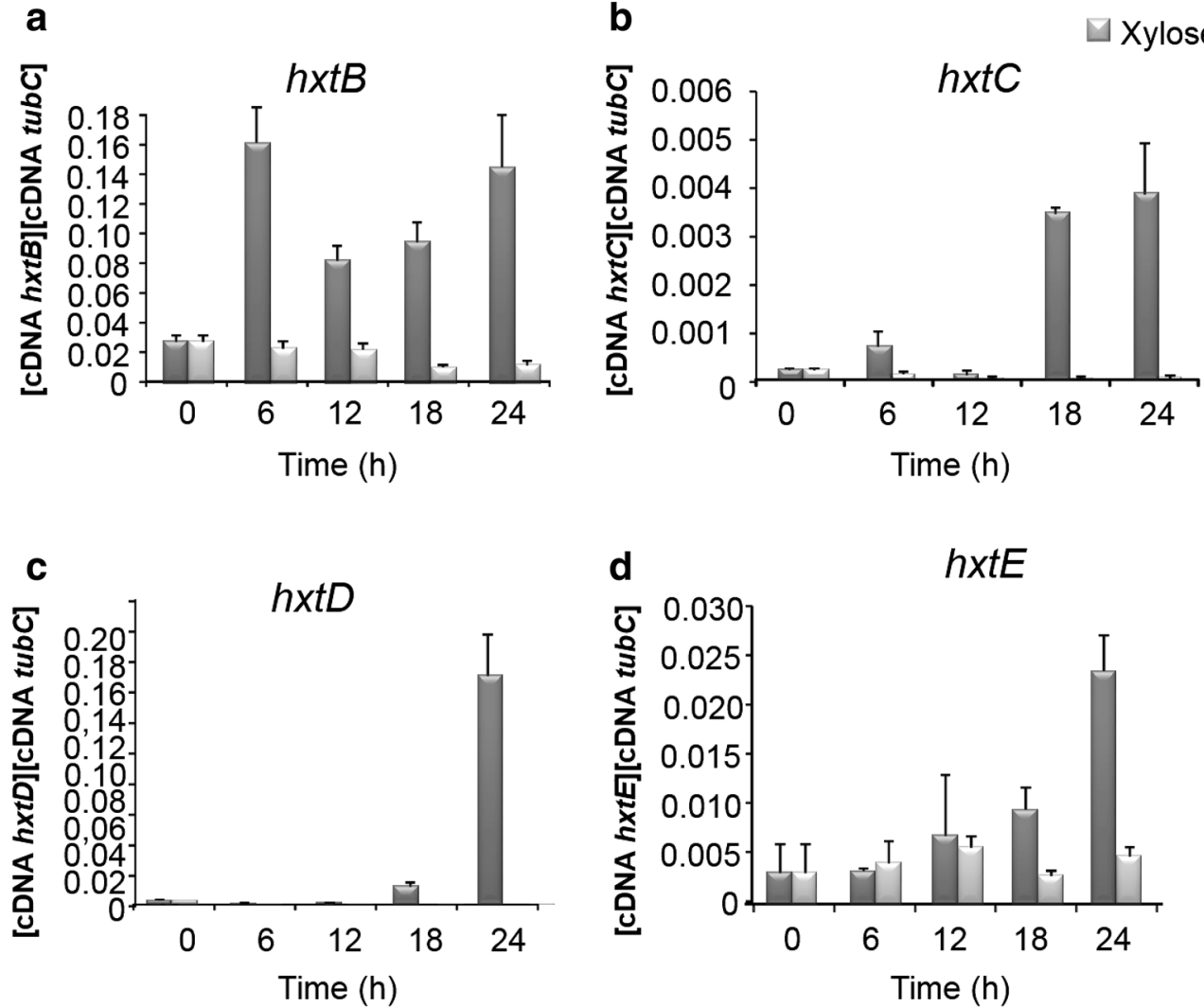

Fig. 4 The expression of the $A$. nidulans hexose transporter-encoding genes $h x t B-E$ is upregulated in the presence of low concentrations of xylose. Transcript levels of $h x t B-E(\mathbf{a}-\mathbf{d})$ were determined by RT-qPCR in the wild-type strain when grown for $0,6,12,18$, and $24 \mathrm{~h}$ in the presence of 0.2 and $2 \%$ xylose. Error bars indicate the standard deviation for three replicates

uptake in S. cerevisiae HxtB::GFP followed single saturation kinetics with a $K_{m}$ value of $0.54 \pm 0.08 \mathrm{mM}$ and a $V_{\max }$ of $1.14 \pm 0.08 \mu \mathrm{m}$ of xylose $\mathrm{h}^{-1}$ per mg cell dry weight (Fig. 7c). In contrast, the $S$. cerevisiae strain which does not contain $h x t B$ was unable to transport xylose (Fig. 7c). In agreement, S. cerevisiae HxtB::GFP was able to grow and consume around $90 \%$ of extracellular xylose after $192 \mathrm{~h}$ of growth in xylose-rich medium and at the same time produce ethanol (Fig. 7d). The control strain, which did not contain the HxtB transporter, did not grow in the presence of xylose and, hence, did not consume xylose and did not produce ethanol (Fig. 7d). In addition, as previously reported, S. cerevisiae strains containing the transporters $\mathrm{HxtB}, \mathrm{HxtC}$, and HxtE are able to grow in the presence of glucose, galactose, fructose, and mannose, while HxtD was not able to use any of these monosaccharides for growth [46]. In contrast, we were not able to see any xylose transport by $S$. cerevisiae strains harboring HxtC, -D, or -E (data not shown). Taken together, these results suggest that HxtB plays, in addition to being a glucose transporter, a major role in xylose uptake.

\section{Discussion}

One of the major drawbacks in biofuel production from lignocellulosic plant material is the inability of fermenting organisms to produce ethanol when growing on sugars other than glucose. Lignocellulose is composed of hexose (glucose) and pentose sugars (mainly xylose) and enzymatic deconstruction of it by, for example, filamentous fungi results in the release of these monosaccharides as well as in the release of oligosaccharides (e.g., the glucose dimer cellobiose) [6]. More specifically, most fermenting organisms are not very efficient at transporting pentose sugars and oligosaccharides into the cell. Complete conversion of all the sugars found in lignocellulose is desired to make $2 \mathrm{G}$ biofuel production an economically feasible process [14]. S. cerevisiae is one of the preferred organisms for fermentation as it is already applied 

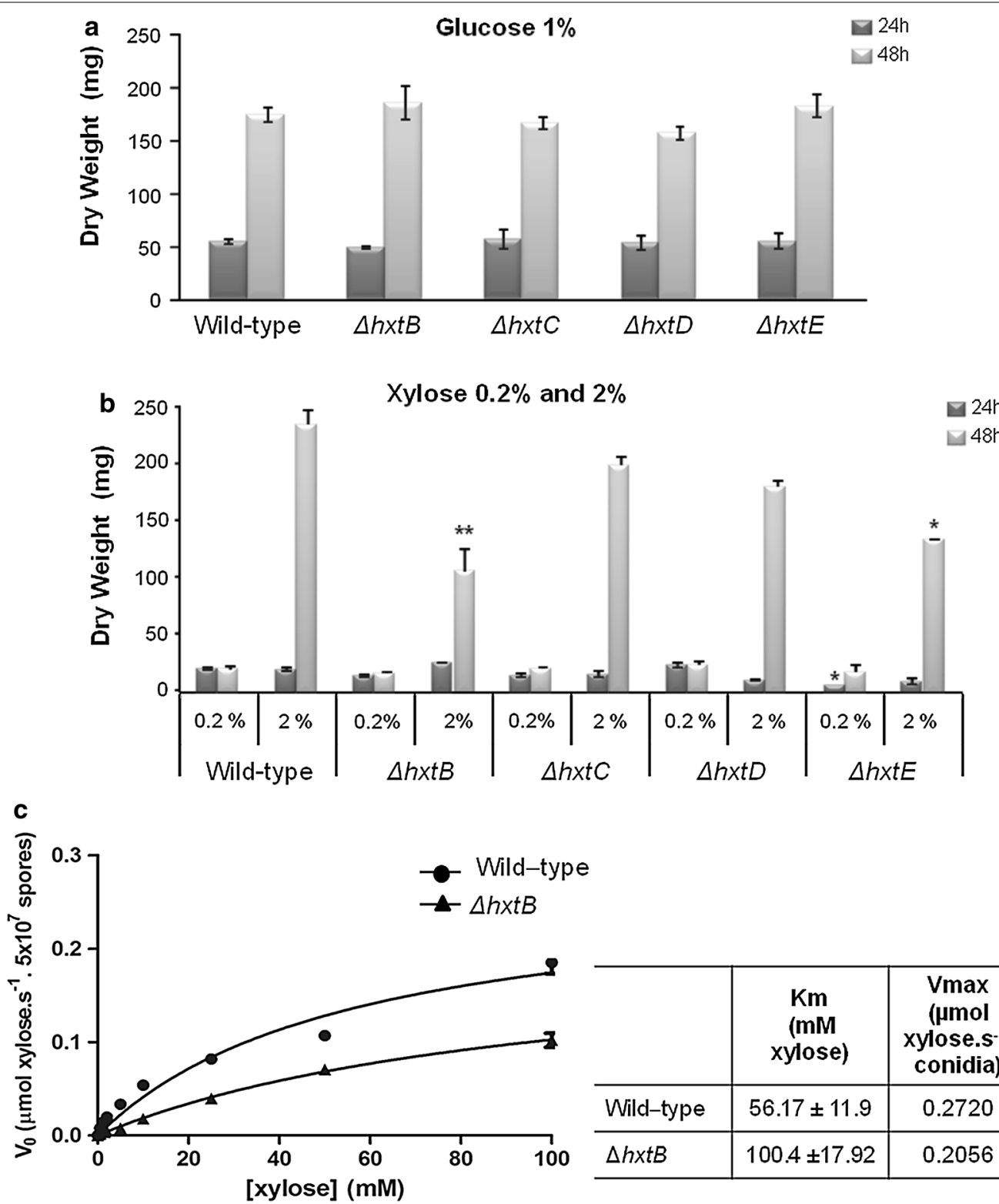

\begin{tabular}{l|c|c}
\hline & $\begin{array}{c}\mathrm{Km} \\
\mathbf{( m M} \\
\text { xylose) }\end{array}$ & $\begin{array}{c}\text { Vmax } \\
(\boldsymbol{\mu m o l} \\
\text { xylose.s } \\
\text { conidia) }^{-1}\end{array}$ \\
\hline Wild-type & $56.17 \pm 11.9$ & 0.2720 \\
\hline$\Delta h x t B$ & $100.4 \pm 17.92$ & 0.2056 \\
\hline
\end{tabular}

Fig. $5 \mathrm{HxtB}$ is a xylose transporter. Fungal dry weight of the wild-type, $\Delta h x t B, \Delta h x t C, \Delta h x t D$, and $\Delta h x t E$ strains was measured after strains were grown for 24 or $48 \mathrm{~h}$ in the presence of $1 \%$ glucose (a) or in the presence of 0.2 and $2 \%$ xylose $(\mathbf{b})$. Xylose transport, as assessed by ${ }^{14} \mathrm{C}-\mathrm{xylose}$ uptake, was measured in the wild-type and $\triangle h x t B$ strains in the presence of different concentrations of xylose (c)

in various industrial processes and is generally regarded as safe [10].

One successful strategy to improve non-glucose uptake in S. cerevisiae is to introduce transporters, from other organisms into its genome. The genomes of filamentous fungi, which are able to internalize a wide variety of mono- and oligosaccharides, are, therefore, screened to find transporters which are able to transport non-glucose sugars. Although this has greatly improved the ability of $S$. cerevisiae to take up pentose sugars such as xylose or cellodextrins such as cellobiose [13, 20, 21, 26], further genetic engineering is required to optimize non-glucose sugar transport. In addition, most transporters encoded by filamentous fungi have not been characterized yet, although these organisms also play a major role in $2 \mathrm{G}$ biofuel production. This work, therefore, aimed at identifying xylose- and cellobiose-specific transporters through screening the genome of the filamentous fungus $A$. nidulans and characterizing them when introduced into $S$. cerevisiae. 
Table 1 Residual xylose in the supernatant during $A$. nidulans growth

\begin{tabular}{llll}
\hline Time (h) & Wild-type & $\boldsymbol{\Delta h x t \boldsymbol { B }}$ & $\boldsymbol{\Delta h \boldsymbol { x t E }}$ \\
\hline $1 \%$ xylose & & & \\
0 & 100 & 100 & 100 \\
24 & $58.7 \pm 6.6$ & $60.7 \pm 2.1$ & $56.5 \pm 2.0$ \\
48 & $23.8 \pm 1.6$ & $49.9 \pm 7.5^{*}$ & $27.8 \pm 0.2$ \\
72 & 0 & $17.4 \pm 2.9^{*}$ & 0 \\
$2 \%$ xylose & & & \\
0 & 100 & 100 & 100 \\
24 & $75.9 \pm 3.7$ & $85.6 \pm 4.2$ & $73.3 \pm 1.6$ \\
48 & $15.4 \pm 4.6$ & $37.3 \pm 0.6^{*}$ & $19.3 \pm 1.2$ \\
72 & 0 & $6.2 \pm 3.4^{*}$ & 0 \\
\hline
\end{tabular}

${ }^{*} p<0.01$

A BLAST analysis of XtrG, encoded by $x \operatorname{tr} G$ and identified as being upregulated in the presence of xylose [33], showed similarity to the N. crassa cellobiose transporter CDT-2. The name of XtrG was subsequently changed to CltA (cellobiose transporter A). Furthermore, another A. nidulans protein, encoded by AN2814, showed high identity to the $N$. crassa cellodextrin transporter CDT-1 and was, therefore, termed CltB. Cellooligosaccharides, such as cellobiose, released during enzymatic degradation of cellulose, have been shown to be important molecules for cellulase gene induction in filamentous fungi, such as T. reese $i, N$. crassa, and P. oxalicum [49-53]. The expression of $c l t A$ increased in the presence of cellobiose. Deletion of $c l t B$ and of $c l t A$ and $c l t B$ simultaneously, but not of $\operatorname{clt} A$, resulted in reduced growth and cellulase secretion in the presence of cellobiose during the first $48 \mathrm{~h}$, although this growth was restored after $72 \mathrm{~h}$ in the $\Delta c l t B$ strain. In contrast, when introduced into $S$. cerevisiae strain SC9721 together with a $\beta$-glucosidaseencoding gene, CltB conferred only slow growth in the presence of different concentrations of cellobiose. These results suggest that the main function of CltB may not be cellobiose transport; it may also function as a transceptor involved in signaling the presence of lignocellulosic biomass. However, we have shown that the introduction of additional functional copies of CltB increases the growth in the presence of low concentrations of cellobiose, strongly indicating CltB is able to transport cellobiose. In $N$. crassa, CDT-1 and CDT-2, in addition to

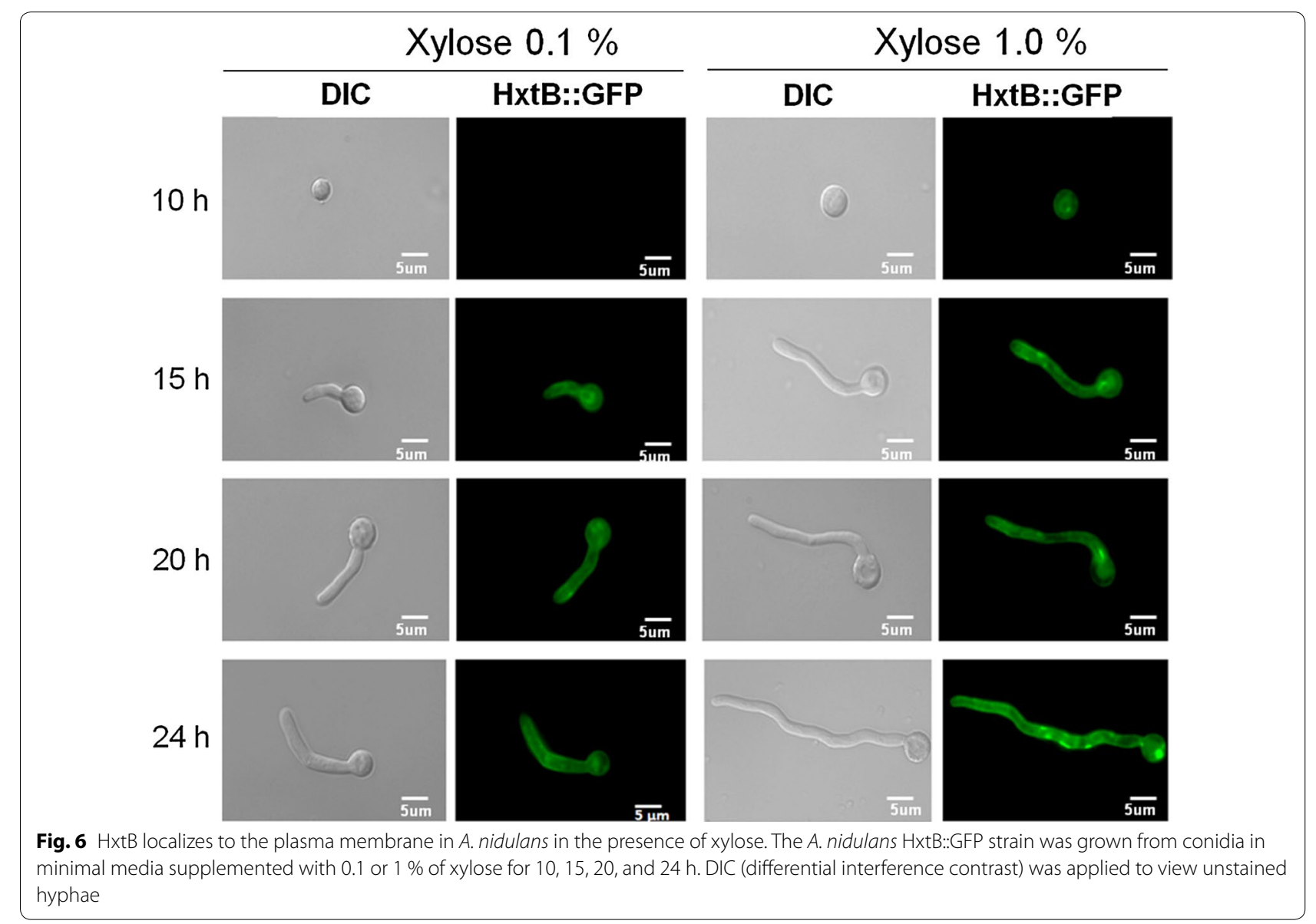




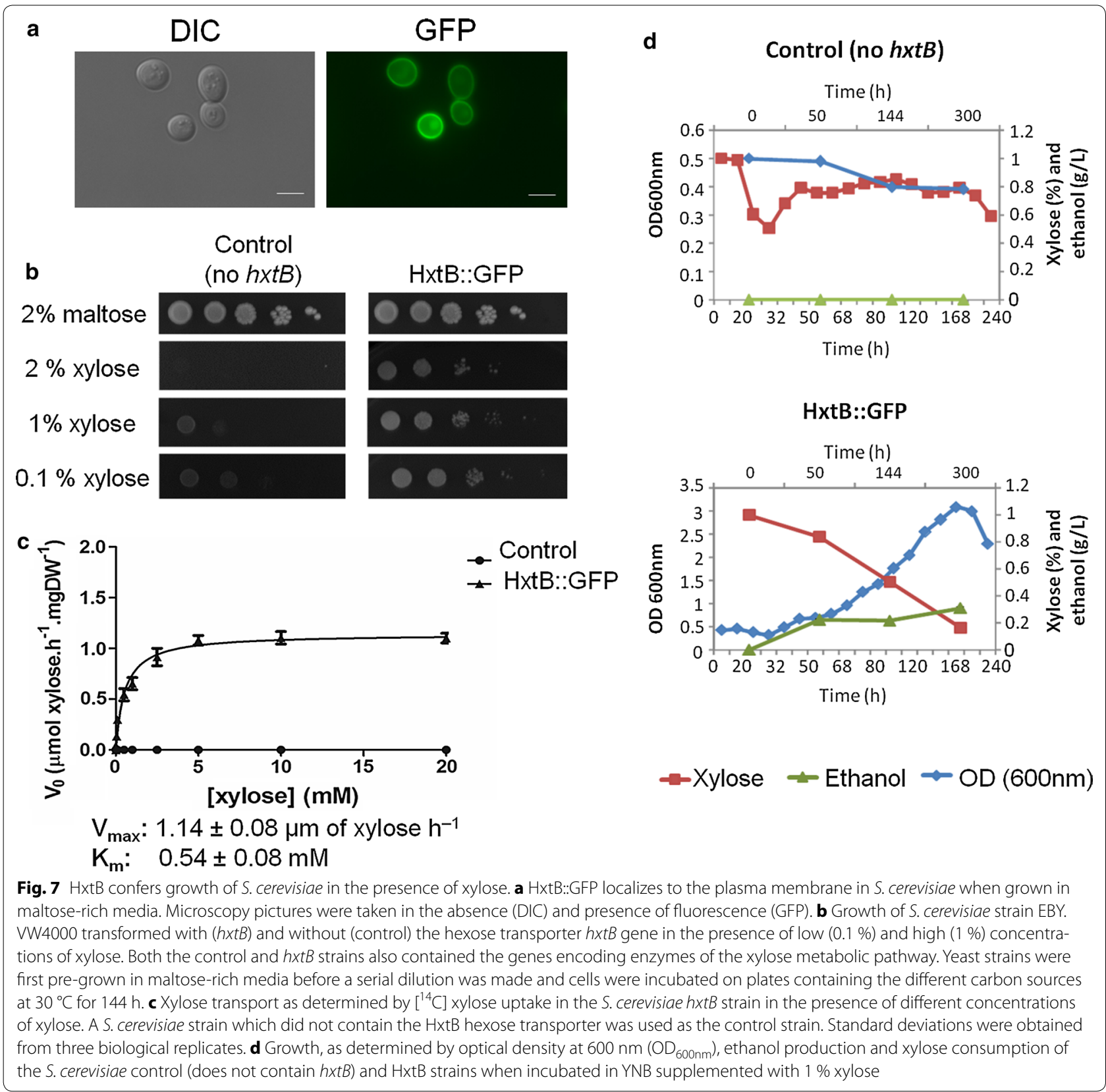

being cellobiose transporters, have been hypothetized to having a role in downstream signaling upon the detection of cellulose by the fungus [42]. Furthermore, the T. reesei transporters STP1 and CRT1 were also proposed to play an important role in the induction of cellulase-encoding genes [32]. This is the first time that a potential transceptor role has been identified for a protein in A. nidulans which is involved in the signaling process of cellulose.

In contrast, deletion of cltA did not result in reduced biomass accumulation and cellulase activity in the presence of cellobiose, but introduction of CltA into $S$. cerevisiae conferred growth in the presence of different concentrations of cellobiose. These results indicate that CltA (formerly XtrG) is a cellobiose transporter and this is the first time that a cellobiose-specific transporter has been identified in $A$. nidulans. The genome of $A$. nidulans encodes 357 MFS transporters and redundancy is very likely to exist between these transporters. This redundancy could compensate for the individual loss of CltA (growth not affected) and CltB (growth restored after $72 \mathrm{~h}$ ), suggesting that other transporters exist with transceptor activities. Deletion of both CltA and CltB had a 
more severe impact on fungal growth in the presence of cellobiose, suggesting that these two proteins do play major roles in cellobiose signaling and uptake and may work together to ensure growth on cellobiose. An intriguing aspect of the biology of $A$. nidulans CltA is why it is also induced in the presence of xylose when it actually is a cellobiose transporter. It is possible that cellobiose transporters could also transport xylooligosaccharides or that the main transcriptional activator of genes encoding proteins required for xylose and xylan metabolism, XlnR (on which CltA was shown to be dependent) can also induce genes encoding cellobiose transporters. Actually, it has already been demonstrated that N. crassa CDT-2 is able to transport both cellodextrins and xylodextrins [54]. Indeed, in $A$. niger, $c b h A$, and $\operatorname{cbh} B$, encoding cellobiohydrolases which catalyze the depolymerization of cellulose were shown to be expressed at much higher levels in the presence of xylose and xylan than when compared to sophorose and cellulose [55]. Furthermore, when grown in the presence of xylan or cellulose, A. nidulans always secretes both cellulases and xylanases (data not shown). This is probably due to the fact that cellulose and hemicelluloses are always found together as they make up the plant cell wall.

Another characteristic of sugar transporters of the MFS is that they very often accept multiple monosaccharides and are, therefore, capable of transporting different sugars into the fungal cells; for example, XtrD transports various monosaccharides [33]. Previously, four glucose transporters were identified in A. nidulans (HxtB-HxtE) and we decided to verify if they could be involved in xylose transport. Deletion of $h x t B$, and to some extent $h x t E$, resulted in significantly reduced growth in the presence of high $(2 \% \mathrm{w} / \mathrm{v})$ concentrations of xylose after $48 \mathrm{~h}$. HxtB (also named MstC) has previously been shown to be a high-affinity glucose transporter which also appears to be able to translocate mannose, galactose, fructose, and xylose [56]. The expression of $h x t B$ is not induced in the presence of glucose but rather in the absence of it, and this gene is also subject to CreA-mediated carbon catabolite repression [56]. This difference in growth and gene expression between high and low concentrations of xylose may therefore be explained by the high-affinity uptake system in which HxtB plays a role or due to the different time points at which both assays were carried out. Furthermore, gene transcription does not necessarily reflect protein secretion and growth. Growth on glucose was not affected, probably, because HxtB is a high affinity glucose transporter which is expressed when glucose is present in low concentrations. Glucose uptake in the presence of high concentrations of this sugar occurs via low affinity transporters such as MstE, which is induced by glucose in $A$. nidulans [57]. Furthermore, deletion of $h x t B$ resulted in decreased affinity for and decreased transport capacity of xylose, indicating that $\mathrm{HxtB}$ is also a xylose transporter in addition to being a glucose transporter. In agreement, when HxtB::GFP was introduced into $S$. cerevisiae, where it located to the plasma membrane, it conferred growth of S. cerevisiae in the presence of different concentrations of xylose and was capable of successfully transporting xylose into the yeast cell. Furthermore, the S. cerevisiae HxtB::GFP strain produced ethanol when growing in xylose-rich media. This work, therefore, identified an $A$. nidulans transporter which in addition to taking up glucose was also efficient at transporting xylose.

Glucose is the preferred carbon source for most microorganisms as it provides rapid energy for survival and niche colonization. Hence, most fungi, including $A$. nidulans are specialized in taking up glucose as soon as it is detected in the environment through high and low affinity uptake systems. As shown in this work, $A$. nidulans transporters have preferentiality for glucose but when this sugar is not available, switch to transporting other sugars, such as xylose, arabinose, or galactose. In addition to the search for transporters which can also translocate pentose or cellodextrins, the focus of research should be directed to the molecular engineering of individual transporters to render them "blind" to glucose and increase affinity and specificity for alternative, non-glucose sugars as was done by $[23,58]$. In addition, yeast strains, which already harbor heterologous introduced transporters, can be genetically modified through directed evolution to improve growth in the presence of pentose sugars or cellodextrins [33]. At the same time, introducing components required for the efficient transport and metabolism of various different sugars into S. cerevisiae, thus allowing the co-fermentation of multiple carbohydrates, has also proved to be a successful strategy $[59,60]$. Furthermore, although the genome of $A$. nidulans (and other filamentous fungi) encodes a multitude of MFS sugar transporters, they have scarcely been characterized and further studies, including those on carbon source sensing and signaling, are required to confirm or reject the above proposed hypothesis. This work identified a cellobiose transporter and a potential cellobiose transceptor in A. nidulans, a role which has also been associated with cellobiose transporters in N. crassa and T. reesei. Furthermore, this study provided further characterization of a glucose/xylose transporter. Taken together, this work provides a preliminary screening and characterization of MFS transporters in A. nidulans and lays a basis for further exploration of sugar sensing and transport in industrially relevant fungi. 


\section{Conclusions}

The knowledge on sugar transport in fungi is very limited, although it presents a key step in the conversion of lignocellulosic biomass to biofuels. In this work, a cellobiose transporter, a xylose transporter, and a putative cellobiose transceptor were identified and characterized in $A$. nidulans. This is the first time that a sensory role for a sugar has been associated to a protein in this fungus. This study, therefore, highlights the importance of continuously screening fungal genomes for transporterencoding genes and in addition, functionally characterizing these proteins. Furthermore, another drawback in the second-generation bioethanol production is the presence of glucose which represses proteins required for the utilization of alternative carbon sources. The identified xylose transporter is also a major glucose transporter, highlighting the preference of $A$. nidulans for this sugar. Furthermore, targeted molecular protein engineering could render these transporters more specific for nonglucose carbon sources. This work, therefore, presents a preliminary basis for further studies which would characterize and engineer known and novel transporters with the aim to introduce them into fermenting yeast strains to successfully convert a large amount of plant cell wall sugars into ethanol.

\section{Methods}

\section{Strains, media, and culture methods}

A list of all the strains used in this work is given in Table 2. All A. nidulans strains were grown at $37{ }^{\circ} \mathrm{C}$ in either liquid (without agar) or solid (with $20 \mathrm{~g} / \mathrm{l}$ agar) minimal medium [MM: $1 \%(\mathrm{w} / \mathrm{v})$ carbon source, $50 \mathrm{ml}$ of a $20 \times$ salt solution $\left(120 \mathrm{~g} / \mathrm{l} \mathrm{NaNO}{ }_{3}, 10.4 \mathrm{~g} / \mathrm{l} \mathrm{KCl}, 30 \mathrm{~g} / \mathrm{l}\right.$ $\left.\mathrm{KH}_{2} \mathrm{PO}_{4}, 10.4 \mathrm{~g} / \mathrm{l} \mathrm{MgSO}{ }_{4}\right), 1 \mathrm{ml}$ of $5 \times$ trace elements $\left(22.0 \mathrm{~g} / \mathrm{l} \mathrm{ZnSO}\right.$, $11 \mathrm{~g} / \mathrm{l}$ boric acid, $5 \mathrm{~g} / \mathrm{MnCl}_{2}, 5 \mathrm{~g} / \mathrm{l}$ $\mathrm{FeSO}_{4}, 1.6 \mathrm{~g} / \mathrm{l} \mathrm{CoCl}_{2}, 1.6 \mathrm{~g} / \mathrm{l} \mathrm{CuSO}_{4}, 1.1 \mathrm{~g} / \mathrm{l}\left(\mathrm{NH}_{4}\right)_{2} \mathrm{MoO}_{4}$, $50 \mathrm{~g} / \mathrm{l}$ ethylenediaminetetraacetic acid (EDTA)] and adjusted to pH 6.5 with $\mathrm{NaOH}$. Depending on the auxotrophy of the strain, uridine $(1.2 \mathrm{~g} / \mathrm{l})$, uracil $(1.2 \mathrm{~g} / \mathrm{l})$ or pyridoxine $(0.005 \mathrm{mg} / \mu \mathrm{l})$ were added. All $S$. cerevisiae strains were grown at $30{ }^{\circ} \mathrm{C}$ in liquid (no agar) or solid (20 g/l agar) YNB medium $(7 \mathrm{~g} / \mathrm{l}$ yeast nitrogen base without amino acids, $0.05 \mathrm{~g} / \mathrm{l}$ histidine, $0.1 \mathrm{~g} / \mathrm{l}$ lysine, $0.1 \mathrm{~g} / \mathrm{l}$ leucine, $0.1 \mathrm{~g} / \mathrm{l}$ tryptophan, $0.1 \mathrm{~g} / \mathrm{l}$ uridine, and $0.1 \mathrm{~g} / \mathrm{l}$ uracil). All reagents were obtained from Sigma Aldrich (St. Louis, MO, USA), except where stated.

\section{Construction of Aspergillus nidulans null mutants}

Standard genetic techniques for A. nidulans strain constructions, transformations, and DNA manipulations were done according to [61]. PCR reactions were performed using Phusion High-Fidelity DNA polymerase (New England Biolabs) or TaKaRa Ex Taq DNA
Polymerase (Clontech USA). A list of all primer pairs can be found in Additional file 4. The gene knock-out strains $\Delta x \operatorname{trF}, \Delta x \operatorname{trG}, \Delta x \operatorname{trH}$, and $\Delta c l t B$ (AN0332, AN8347, AN9173 and AN2814, respectively) were obtained through replacing each gene with a prototrophic marker gene. Gene replacement cassettes were generated by in vivo recombination in $S$. cerevisiae as previously described by [62]. Briefly, the $5^{\prime}$ UTR of each target gene was PCR amplified using specific primers: $x$ trF (primers P1 and P2), $x \operatorname{trG}$ (primers P7 and P8), $x \operatorname{trH}$ (primers P13 and P14), and cltB (primers P27 and P28). Similarly, the $3^{\prime} \mathrm{UTR}$ regions of each gene were amplified by PCR: $x$ trF (primers $\mathrm{P} 3$ and $\mathrm{P} 4), x \operatorname{trG}$ (primers $\mathrm{P} 9$ and $\mathrm{P} 10), x \operatorname{trH}$ (primers P15 and P16), and cltB (primers P29 and P30). Pyridoxine or uridine/uracil were used as prototrophic markers, and their respective genes (pyro $A$ and pyrG) were amplified by PCR from plasmids pAFpyro (primers P37 and P38) and pCDA21 (primers P35 and P36), respectively (Table 2$)$. The individual gene fragments $\left(5^{\prime}\right.$ and $3^{\prime}$ UTRs and prototrophic marker gene) were transformed, together with plasmid pRS426, which was linearized with EcoRI and BamHI, into S. cerevisiae SC9721 using the lithium acetate method [63]. Positive S. cerevisiae transformation candidates were grown in YNB-URA medium, before gDNA was extracted and PCRs were run to confirm the correct construction. The cassettes were then PCR-amplified from S. cerevisiae genomic DNA, purified and used to transform A. nidulans TN02A3 strain, according to [64]. Positive A. nidulans transformation candidates were selected and purified through three rounds of growth on plates and gDNA was extracted. Gene deletions were confirmed by Southern blots (Additional file 5).

To construct the complemented strain $\Delta c l t B:: c l t B^{+}$, the complementing cassette containing the $5^{\prime}$ UTR region plus the cltB gene and the $3^{\prime}$ UTR region was amplified by PCR from $A$. nidulans genomic DNA using specific primers (P27 and P30). The A. nidulans $\triangle c l t B \mathrm{Ku} 80^{+}$ mutant was co-transformed with pCDA21 plasmid and the $\mathrm{cltB}^{+}$complementing cassette. Positive $A$. nidulans complemented candidates were selected and purified through three rounds of growth on plates, gDNA was extracted, and the candidates were confirmed by PCR (Additional file 6).

\section{Construction of Aspergillus nidulans GFP- tagged strains}

All $A$. nidulans GFP-tagged strains were constructed as described in the previous section ("Construction of A. nidulans null mutants") with the exception that genes were not replaced by prototrophic markers but were instead C-terminally tagged with GFP. The selective marker gene pyrG was also introduced. A list of all primers used for strain constructions can be found in 
Table 2 Strains and plasmids used in this work

\begin{tabular}{|c|c|c|}
\hline Strains/plasmids & Genotype & Reference \\
\hline \multicolumn{3}{|l|}{ S. cerevisiae } \\
\hline EBY.VW4000 & 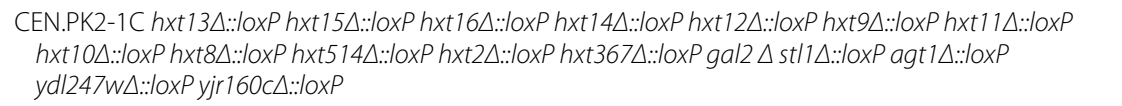 & {$[48]$} \\
\hline SC9721 & MATa his 3-D200 URA 3-52 leu2D1 lys 2D202 trp 1D63 & FGSC \\
\hline EBY.VW4000 +pRH195m +pRH274 & EBYVW4000 pRH195 pRH274 & {$[33]$} \\
\hline hxtBGFP EBY.VW4000 & EBYVW4000 pRH195 hxtB pRH274 & This work \\
\hline xtrF::GFP EBY.VW4000 & EBYVW4000 pRH195 xtrF pRH274 & This work \\
\hline xtrG::GFP EBY.VW4000 & EBYVW4000 pRH195 xtrG pRH274 & This work \\
\hline xtrH::GFP EBY.VW4000 & EBYVW4000 pRH195 xtrH pRH274 & This work \\
\hline cltB::GFP EBY.VW4000 & EBYVW4000 pRH195 cltB pRH274 & This work \\
\hline SC9721 cltA::GFP gh1-1 & SC9721 pRH195 cltA pGH1 & This work \\
\hline SC9721 cltB::GFP gh1-1 & SC9721 pRH195 cltB pGH1 & This work \\
\hline SC9721_pGH1 & SC9721 pGH1 & This work \\
\hline SC9721_pCDT-1 gh1-1 & SC9721 pCDT-1 pGH1 & This work \\
\hline \multicolumn{3}{|l|}{ A. nidulans } \\
\hline TN02A3 & pyroA4 pyrG89; chaA1; $\triangle n K u A:: \arg B$ & {$[70]$} \\
\hline HxtB::GFP TN02A3 & pyrG89; pyroA4; $\triangle$ nku70::argB; hxtB::GFP::.pyrG & This work \\
\hline HxtE::GFPTN02A3 & pyrG89; pyroA4; $\triangle$ nku70::argB; hxtE::GFP::pyrG & This work \\
\hline$\Delta x t r F$ & pyroA4 pyrG89; ChaA1; $\triangle n K u A:: a r g B ; \triangle x t r F:: p y r G$ & This work \\
\hline$\Delta x \operatorname{trG} / \Delta c l t A$ & pyroA4 pyrG89; ChaA1; $\triangle n K u A:: a r g B ; \triangle x \operatorname{trG}:$. pyrG & This work \\
\hline$\Delta x t r H$ & pyroA4 pyrG89; chaA1; $\triangle n K u A:: \arg B ; \triangle x t r H:: p y r G$ & This work \\
\hline$\Delta c l t B$ & pyroA4 pyrG89; chaA1; $\triangle n K u A:: a r g B ; \triangle A l t B:: p y r o A 4$ & This work \\
\hline$\Delta h \times t B$ & pyroA4 pyrG89; chaA1; $\triangle n K u A .:$ argB; $\triangle$ hxtB.:pyroA4 & {$[46]$} \\
\hline$\triangle h x t E$ & pyroA4 pyrG89; chaA1; $\triangle n K u A .: \operatorname{argB;} \triangle 4 x x t E .: p y r o A 4$ & {$[46]$} \\
\hline$\triangle C l t A \triangle C l t B$ & pyroA4 pyrG89; chaA1; $\triangle$ nKuA::argB; $\triangle$ cltA::pyrG89; $\triangle$ cltB::pyroA4 & This work \\
\hline GR5 & wA1 pyroA1pyrG89 & FGSC \\
\hline$\triangle C l t B: .: c l t B^{+}$ & $\triangle \mathrm{clt} B: .: \mathrm{clt} B^{+}: . p y r G^{+}$pyrG89 & This work \\
\hline oCltB3 & wA1 pyroA1 pyrG89 cltB3::gfp::pyrG ${ }^{+}$ & This work \\
\hline \multicolumn{3}{|l|}{ Plasmids } \\
\hline $\mathrm{pRH} 195^{\mathrm{a}}$ & pBluescript II SK+, TRP1, CEN6, ARSH4+PHXT7-XKS1-THXT7 & {$[24]$} \\
\hline $\mathrm{pRH} 274$ & pBluescript II SK+, URA3, CEN6, ARSH4 + PPGK1-XYL1-TPGK1; PADH1-XYL2-TADH1; PHXT7-XKS1-THXT7 & {$[66]$} \\
\hline pRS426 & ampR lacZ URA3 & {$[65]$} \\
\hline pCDA21 & Zeo::pyr ampR & [71] \\
\hline pGH1-1 & pRS425 PGK1p-gh1-1-CYC1t & {$[26]$} \\
\hline pCDT-1 & pRS426 PGK1p-cdt-1-CYC1t & {$[26]$} \\
\hline
\end{tabular}

a The original vector pRH195 carries the XKS1 gene which was released after digestion with Spel and Sall. The resultant vector without the XKS1 gene was used in this work for compl ementation assays

Additional file 4. The xtrF-H, hxtB and hxtE genes were amplified by PCR using primers P5/P6, P11/P12, P17/ P18, P42/P43, and P48/P49, respectively. The 3 ' UTRs of genes $x \operatorname{tr} F-H, h x t B$, and $h x t E$ constructions were amplified by PCR using primers P3/P4 (xtrF), P9/P10 ( $x$ trG), P15/P16 ( $x t r H), \mathrm{P} 30 / \mathrm{P} 32$ (cltB), P44/P45 (hxtB), and P50/ P51 $(h x t E)$. The $g f p$ gene was separated from the target gene by four additional codon triplets that after translation produce a four amino acid residue linker (glycinethreonine-arginine-glycine) region termed Spacer-GFP [65]. To allow fusion of GFP to our protein of interest, the stop codon of the gene ORF was removed when designing the primers. The GFP was amplified from the pMCB17apx plasmid (kindly provided by Vladimir P. Efimov) with primers P39/P40. The pyrG gene was amplified as described above. GFP-tagged gene constructions were confirmed by PCR in A. nidulans.

In addition, the cltB overexpression strain (GR5 CltB::GFP strain) was constructed in the $A$. nidulans GR5 background, because this strain allows multiple nonhomologous ectopic integrations. Again, the $A$. nidulans GFP-tagged strains were constructed as described 
in the previous section ("Construction of A. nidulans null mutants") with the exception that genes were not replaced by prototrophic markers but were instead C-terminally tagged with GFP and the selective marker gene pyrG was also introduced. The cltB gene (primers P27/ P31) and the $3^{\prime}$ UTR (primers P30/P32) were amplified by PCR. The multiple integrations of CltB::GFP cassette were confirmed by Southern blot (Additional file 1).

For all constructions above described, the $g f p$ gene was separated from the target gene by four additional codon triplets that after translation produce a four amino acid residue linker (glycine-threonine-arginine-glycine) region termed Spacer-GFP [65]. To allow fusion of GFP to our protein of interest, the stop codon of the gene ORF was removed when designing the primers. The GFP was amplified from the pMCB17apx plasmid (kindly provided by Vladimir P. Efimov) with primers P39/P40. The pyrG gene was amplified as described above. GFPtagged gene constructions were confirmed by PCR in $A$. nidulans or southern blot. The GFP was amplified from the pMCB17apx plasmid (kindly provided by Vladimir P. Efimov) with primers P39/P40. The $p y r G$ gene was amplified as described above. For $x t r F-H, h x t B$ and $h x t E$ mutants, GFP-tagged gene constructions were confirmed by PCR in $A$. nidulans.

\section{Construction of Saccharomyces cerevisiae strains}

Strain EBY.VW4000 (Table 2) was used for the Saccharomyces cerevisiae complementation assays [48]. A list of all primers can be found in Additional file 4. The $x \operatorname{trF}-H$, cltB, and $h x t B$ ORFs were amplified by PCR from cDNA obtained from $A$. nidulans strains using primers P19/P20 ( $x t r F)$, P21/P22 ( $x t r G)$, P23/P24 ( $x t r H)$, P33/P34 (cltB), and P46/P47 ( $h x t B)$, respectively. Plasmid pRH195 was double digested with SpeI and SalI for linearization and release of the XKS1 gene (generating the pRH195 m). For in vivo recombination, plasmid pRH195 $\mathrm{m}$ was transformed into S. cerevisiae EBY.VW4000, which already contained plasmid pRH274 [33], together with all the PCR-amplified sugar transporters and GFP fragments using the lithium acetate method [63]. The $g f p$ gene was amplified from plasmid pMCB17apx using primers P25/P26. S. cerevisiae EBY.VW4000 is unable to metabolize xylose and in addition to being transformed with the $A$. nidulans transporter-encoding genes, it was also transformed with genes encoding enzymes of the xylose metabolic pathway. Saccharomyces stipitis xylose reductase (XR) and xylose dehydrogenase $(\mathrm{XDH})$ as well as $S$. cerevisiae xylulose kinase (XK) were introduced in EBY.VW4000 via plasmid pRH274 (Table 2), where the three enzyme-encoding genes were placed under the control of the PGK1, ADH1, and $H X T 7$ constitutive promoters, respectively [66]. Transformants were selected for tryptophan and uridine prototrophy on solid YNB lacking both tryptophan and uridine and supplemented with $2 \%$ maltose.

Saccharomyces cerevisiae SC9721 strain was used to construct the yeast strains expressing the cellobiose transporters $c l t A$ and $c l t B$. The SC9721 strain was first transformed with the pGH1 plasmid which contains the $\beta$-glucosidase-encoding gene gh1-1 from $N$. crassa [26]. The $c l t A$ and $c l t B$ cellobiose transporter genes were amplified from cDNA of $A$. nidulans using primers P52/ P22 (cltA) and P53/P34 (cltB). Plasmid pRH195 m was used to transform $S$. cerevisiae with the respective transporter genes. Furthermore, S. cerevisiae strain SC9721 was also transformed with plasmid pCDT-1, containing the already characterized $N$. crassa $c d t-1$ cellobiose transporter-encoding gene, which was used as a positive control in ours assays [12]. All S. cerevisiae transformations were carried out using the lithium acetate method [63] and strain constructions were confirmed by PCR.

\section{gDNA extraction from A. nidulans and S. cerevisiae}

Genomic DNA extractions of A. nidulans and S. cerevisiae were performed according to [67] and [63].

\section{Microscopy}

A. nidulans strains HxtB::GFP, HxtE::GFP, XtrF::GFP, XtrG::GFP, XtrH::GFP, and CltB::GFP were grown from spores in $3 \mathrm{ml}$ of $\mathrm{MM}$ containing 0.1 and $1 \%$ xylose for $10,15,20$, and 24 at $30^{\circ} \mathrm{C}$ in a small Petri dish containing a microscopy cover slip. The oCltB3::GFP strain was previously inoculated $16 \mathrm{~h}$ at $30{ }^{\circ} \mathrm{C}$ in a small Petri dish containing a microscopy coverslip and $3 \mathrm{~mL}$ of MM supplemented with $1 \%$ fructose as a carbon source. After $16 \mathrm{~h}$, the germlings were washed with $1 \mathrm{X}$ PBS and transferred to 0.5 and $1 \%$ cellobiose for 4 or $8 \mathrm{~h}$. Coverslips were washed with $1 \times$ PBS $(137 \mathrm{mM}$ sodium chloride, $2.7 \mathrm{mM}$ potassium chloride, $10 \mathrm{mM}$ disodium hydrogen phosphate, $1.8 \mathrm{mM}$ potassium dihydrogen phosphate) and viewed under the microscope. S. cerevisiae EBY.VW400 strain harboring the $h x t B, x t r F-H$, and $c l t B$ tagged to GFP constructions was grown $48 \mathrm{~h}$ in $0.5 \mathrm{ml}$ of liquid YNB-trp-ura medium supplemented with $2 \%$ maltose for $24 \mathrm{~h}$ at $30{ }^{\circ} \mathrm{C}$ in a 24-wells plate. Cells were washed with PBS and viewed under the microscope. All slides were viewed with a Carl Zeiss (Jena, Germany) microscope using the $100 \times$ magnification oil immersion objective lens (EC Plan-Neofluar, NA 1.3) equipped with a 100-W HBO mercury lamp epifluorescence module. Phase contrast brightfield and fluorescent images were taken with an AxioCam camera (Carl Zeiss), and images were processed using the AxioVision software version 3.1 and saved as TIFF files. Further processing was performed using Adobe Photoshop 7.0 (Adobe Systems Incorporated, CA). 


\section{Dry weight measurement}

A total of $5 \times 10^{7}$ spores of $A$. nidulans wild-type (TN02A3) and mutant strains ( $\triangle x t r F-H$ and $\Delta h x t B)$ were inoculated in $50 \mathrm{ml}$ of liquid minimal medium supplemented with $1 \%$ glucose, $2 \%$ xylose or $0.2 \%$ xylose. $2.5 \times 10^{7}$ spores of $A$. nidulans wild-type (TN02A3) and mutant strains $(\triangle c l t A, \Delta c l t B$ and $\triangle c l t A \Delta c l t B)$ were inoculated in $50 \mathrm{~mL}$ of liquid minimal medium supplemented with $1 \%$ glucose or $1 \%$ cellobiose. Strains were grown for 24 and $48 \mathrm{~h}$ in xylose-rich media and for 48 and $72 \mathrm{~h}$ in cellobiose-rich media at $37^{\circ} \mathrm{C}, 180 \mathrm{rpm}$. Mycelia were harvested by vacuum filtration, snap-frozen in liquid $\mathrm{N}_{2}$, freeze-dried and subsequently weighed.

\section{RNA extraction and real-time $P C R$ reactions}

To measure the expression of $x t r F-H$, a total of $10^{7}$ spores from the $A$. nidulans wild-type, creAd30 or $\Delta x \ln R$ strains were inoculated in $50 \mathrm{ml}$ of liquid $\mathrm{MM}$ containing $1 \%$ fructose for $16 \mathrm{~h}$ at $37^{\circ} \mathrm{C}, 180 \mathrm{rpm}$. Mycelia were washed with sterile water and transferred to MM supplemented with $1 \%$ xylose or $1 \%$ xylose and $1 \%$ glucose for 6,12 and $24 \mathrm{~h}$ at $37^{\circ} \mathrm{C}, 180 \mathrm{rpm}$. Alternatively, $10^{7}$ spores from the wild-type $A$. nidulans strain was inoculated in $50 \mathrm{ml}$ of liquid MM supplemented with $1 \%$ glucose, $1 \%$ sorbitol, $1 \%$ xylose, $1 \%$ fructose, $1 \%$ maltose, $1 \%$ galactose, and $1 \%$ mannose at $37{ }^{\circ} \mathrm{C}, 180 \mathrm{rpm}$ for 8 or $16 \mathrm{~h}$. All mycelia were harvested by vacuum filtration, snap-frozen in liquid $\mathrm{N}_{2}$, and stored at $-80^{\circ} \mathrm{C}$.

To measure the expression of $\operatorname{clt} A$ and $\operatorname{clt} B$, a total of $10^{7}$ spores from the $A$. nidulans wild-type strain were inoculated in $50 \mathrm{ml}$ of liquid $\mathrm{MM}$ containing $1 \%$ fructose for $16 \mathrm{~h}$ at $37^{\circ} \mathrm{C}, 180 \mathrm{rpm}$. Mycelia were washed with sterile water and transferred to MM supplemented with $1 \%$ cellobiose for additional 1,2 , and $4 \mathrm{~h}$ at $37^{\circ} \mathrm{C}, 180 \mathrm{rpm}$. Mycelia were harvested by vacuum filtration, snap frozen in liquid $\mathrm{N}_{2}$, and stored at $-80^{\circ} \mathrm{C}$. clt $\mathrm{A}$ and cltB

Mycelia were ground to a fine powder under liquid $\mathrm{N}_{2}$, and RNA was extracted using Trizol (Invitrogen, Carlsbad, CA, USA), according to manufacturer's instructions. The quality of the RNA $(10 \mu \mathrm{g})$ was checked by running them through the Bioanalyzer. RNA samples were DNAse-treated as previously described by [67], purified with the RNeasy ${ }^{\circledR}$ Mini Kit (Qiagen, Valencia, CA, USA) and quantified on the NanoDrop ${ }^{\circledR} 2000$ (Thermo Scientific) machine. RNA integrity was confirmed using the Bioanalyser Nano Kit (Agilent Technologies) and the Agilent Bioanalyser 2100, using an RIM value of 6.0 as a threshold.

RNA was then reverse transcribed to cDNA using the Superscript III Reverse transcriptase kit (Invitrogen), according to manufacturer's instructions. All RT-qPCR reactions were performed using the ABI 7500 Fast RealTime PCR System (Applied Biosystems, Foster City, CA,
USA) and the SYBR Green PCR Master Mix kit (Applied Biosystems), according to manufacturer's instructions. Reactions and calculations were performed as previously described [68]. All primers are listed in Additional file 4.

\section{Xylose uptake assay}

Xylose uptake rates were measured by monitoring the incorporation of D-[U- $\left.{ }^{14} \mathrm{C}\right]$ xylose $[289.0 \mathrm{mCi} / \mathrm{mmol}$ $(10.693 \mathrm{GBq}) / \mathrm{mmol}$ (Perkin Elmer Life Sciences) into germinating conidia in the presence of various D-xylose concentrations according to [33] with modifications. A total of $1.2 \times 10^{9} \Delta h x t B$ conidia were inoculated in $600 \mathrm{ml} \mathrm{MM}$ containing $1 \%$ glycerol $(\mathrm{w} / \mathrm{v})$ for $5 \mathrm{~h}$ at $37{ }^{\circ} \mathrm{C}, 180 \mathrm{rpm}$. Swollen conidia were harvested by vacuum filtration through nitrocellulose filters (Fisherbrand) and washed twice with ice-cold water. Conidia were re-supended in water to get a concentration of $2.5 \times 10^{7}$ conidia/ $250 \mu \mathrm{l}$. A total of $2.5 \times 10^{7}$ spores were inoculated with different concentrations of D-xylose $(0.1-100 \mathrm{mM})$ in $1.5 \mathrm{ml}$ tubes together with $1 \mu \mathrm{l}$ of radiolabelled ${ }^{14} \mathrm{C}$-xylose $(0.2 \mu \mathrm{Ci})$ and incubated at room temperature for 30-60 s. Xylose uptake was stopped by adding $1.5 \mathrm{ml}$ ice-cold water and conidia were immediately harvested by vacuum filtration through nitrocellulose filters. Conidia were washed again two times with $1.5 \mathrm{ml}$ ice-cold water.

Saccharomyces cerevisiae HxtB::GFP EBY.VW400 strain was inoculated in $300 \mathrm{ml}$ of YNB medium supplemented with $2 \%$ maltose until they reached the exponential growth phase $\left(\mathrm{OD}_{600 \mathrm{~nm}}\right.$ of 0.6). Yeast cells were pelleted by centrifugation at $4000 \mathrm{rpm}$ for $5 \mathrm{~min}$, washed twice with $50 \mathrm{ml}$ ice-cold water, and then re-suspended in $4.5 \mathrm{ml}$ of ice-cold water. $40 \mu \mathrm{l}$ of this cell suspension were transferred to $1.5 \mathrm{ml}$ Eppendorf tubes which were then incubated at $30{ }^{\circ} \mathrm{C}$ for $5 \mathrm{~min}$ for temperature equilibration. $10 \mu \mathrm{l}$ of different concentrations of xylose ( 0.1 to $100 \mathrm{mM}$ xylose) and $0.2 \mu \mathrm{Ci}$ of ${ }^{14} \mathrm{C}$-xylose were added to the yeast cells. Xylose uptake was allowed to proceed for $10 \mathrm{~s}$ through vigorous vortexing before the reaction was stopped through the addition of $1.5 \mathrm{ml}$ ice-cold water. Cells were harvested by vacuum filtration through nitrocellulose filters and washed two times with ice-cold water.

All nitrocellulose filters containing the fungal cells were transferred to $3 \mathrm{ml}$ of ScintiSafeTM Econo1 scintillation liquid (Fisher Scientific), and the D-[U- $\left.{ }^{14} \mathrm{C}\right]$ xylose taken up by the cells was measured using the Tri-Carb ${ }^{\circledR}$ 2100TR Liquid Scintillation Counter.

\section{Assaying extracellular xylose concentrations}

The EBY.VW4000 + pRH195 m + pRH274 (control) and the HxtB::GFP yeast strains were inoculated (initial $\left.\mathrm{OD}_{600} 0.5\right)$ in $50 \mathrm{ml} \mathrm{YNB-trp-ura} \mathrm{medium} \mathrm{supplemented}$ with $1 \%(\mathrm{w} / \mathrm{v})$ xylose at $30^{\circ} \mathrm{C}, 150 \mathrm{rpm}$ for $300 \mathrm{~h}$. At each 
time point, $2.0 \mathrm{ml}$ of the culture was collected, centrifuged, and the supernatants were stored at $-80{ }^{\circ} \mathrm{C}$. The xylose concentration in the supernatants was measured using the D-xylose assay kit (Megazyme) following manufacturer's instructions. Absorbance was measured at $340 \mathrm{~nm}$ in a 96-well polystyrene plate (Corning) using the SpectraMax I3 spectrometer (molecular devices).

\section{Assaying extracellular ethanol concentrations}

The EBY.VW4000 + pRH195 m + pRH274 (control) and HxtB::GFP yeast strains were inoculated (initial $\mathrm{OD}_{600}$ $0.5)$ in $50 \mathrm{ml}$ YNB-trp-ura medium supplemented with $1 \%(\mathrm{w} / \mathrm{v})$ xylose at $30^{\circ} \mathrm{C}, 150 \mathrm{rpm}$ for $300 \mathrm{~h}$. At each time point, $2.0 \mathrm{ml}$ of the culture was collected, centrifuged, and the supernatants were stored at $-80^{\circ} \mathrm{C}$. Ethanol production was determined by measuring the absorption of NADH at $340 \mathrm{~nm}$ as previously described [69] with modifications. Reactions were started through mixing $100 \mu \mathrm{l}$ assay buffer $(50 \mathrm{mM}$ pyrophosphate, $50 \mathrm{mM}$ semicarbazide, and $20 \mathrm{mM}$ glycine, $\mathrm{pH} 8.8$ ) with $0.643 \mathrm{mM}$ $\mathrm{NAD}^{+}, 5 \mathrm{U}$ alcohol dehydrogenase and $10 \mu \mathrm{l}$ sample supernatant in a 96-well polystyrene plate (Corning). Samples were incubated at $30^{\circ} \mathrm{C}$ for $5 \mathrm{~min}$, and then, the ethanol concentration was measured at $340 \mathrm{~nm}$ using the SpectraMax I3 spectrometer (Molecular devices).

\section{Growth of Saccharomyces cerevisiae strains on solid medium}

S. cerevisiae strains were inoculated in $50 \mathrm{ml} \mathrm{YNB}$ medium supplemented with $2 \%$ maltose or $1 \%$ glucose for $24 \mathrm{~h}$ at $30{ }^{\circ} \mathrm{C}, 150 \mathrm{rpm}$ until an $\mathrm{OD}_{600 \mathrm{~nm}}$ of 0.1 . Yeast cells were centrifuged at $4.000 \mathrm{rpm}$ for $5 \mathrm{~min}$, washed two times with water, and re-suspended in water to a final concentration of 1.0 at $\mathrm{OD}_{600 \mathrm{~nm}}$. A serial dilution of 1:10 of the yeast cells was made, and $5 \mu \mathrm{l}$ of the cell suspensions were spotted on plates containing $1 \%$ glucose, $0.1,1$ or $2 \%$ cellobiose. Plates were incubated at $30{ }^{\circ} \mathrm{C}$ for $168 \mathrm{~h}$.

\section{Yeast growth rates}

The SC9721 cltA::GFP gh1-1, SC9721 cltB::GFP gh1-1 and SC9721 pCDT-1 gh1-1 strains were grown in YNB medium supplemented with $1 \%$ glucose for $24 \mathrm{~h}$ at $30{ }^{\circ} \mathrm{C}$, $150 \mathrm{rpm}$. The OD at $600 \mathrm{~nm}$ was measured; the cell cultures were diluted to $\mathrm{OD}_{600 \mathrm{~nm}}$ of 0.1 and transferred to $50 \mathrm{ml}$ YNB medium supplemented with the respective carbon source (glucose $1 \%$ or cellobiose $0.1-2 \%$ ) at $30{ }^{\circ} \mathrm{C}, 150 \mathrm{rpm}$ for $144 \mathrm{~h}$. The $\mathrm{OD}_{600 \mathrm{~nm}}$ was measured periodically at the indicated time points.

\section{Cellulase assays}

A total of $10^{7}$ spores from wild-type, $\Delta c l t A, \Delta c l t B$ and $\triangle c l t A \Delta c l t B$ mutant strains were inoculated in liquid
MM supplemented with $1 \%$ fructose at $37{ }^{\circ} \mathrm{C}, 180 \mathrm{rpm}$ for $16 \mathrm{~h}$. Mycelia were washed with sterile water and transferred to MM supplemented with $1 \%$ Avicel $^{\mathrm{TM}}$ for 5 days at $37{ }^{\circ} \mathrm{C}, 180 \mathrm{rpm}$. The supernatant was separated from the mycelia using miracloth. Cellulase (endo-1,4$\beta$-glucanase) activity in the supernatants was measured using Azo-CM-Cellulose (Megazyme International, Bray, Ireland) as a substrate, according to manufacturer's instructions.

\section{Additional files}

Additional file 1. Construction of an overexpressing CltB::GFP mutant strain. (A) Genomic DNA from A. nidulans wild-type GR5 and CltB::GFP transformant strains were isolated and cleaved with the enzyme Pstl; a 2.7-kb DNA fragment from the $5^{\prime}$-noncoding region plus the $c$ ttB gene was used as a hybridization probe. This fragment recognizes a single DNA band (about $5.0 \mathrm{~kb}$ ) in the wild-type strain and a single DNA band (about $3.0 \mathrm{~kb}$ ) in the CItB::GFP homologously integrated cassette. Different size of bands indicates the multiple integration of the GFP cassette. (B) Southern blot.

Additional file 2. Enzymatic kinetics. (A) Eadie-Hofstee and (B) Lineweaver-Burk plots for the data of the Fig. $6 \mathrm{c}$.

Additional file 3. HxtE is target to the plasma membrane in A. nidulans in the presence of xylose. The A. nidulans HxtE::GFP strain was grown from conidia in minimal media supplemented with $0.1 \%$ or $1 \%$ of xylose for $10 \mathrm{~h}, 15 \mathrm{~h}, 20 \mathrm{~h}$, and $24 \mathrm{~h}$. DIC (differential interference contrast) was applied to view unstained hyphae.

Additional file 4. Primers used in this work.

Additional file 5. Genomic DNA from the A. nidulans wild-type, $\triangle x \operatorname{tr} G$ (AN8347), $\Delta x \operatorname{trH}$ (AN9173), $\Delta c l t B$ (AN2814) and the double $\Delta c / t A \Delta c l t B$ strains was extracted and digested with different restriction enzymes to confirm the deletion strains. Diagram (A.) and Southern blot (B.) of the wild-type and $\triangle x \operatorname{trG}$ strains when digested with Sacl. A 1-kb DNA fragment from the $x \operatorname{trG} 3^{\prime} U T R$ (untranslated) region was used as a hybridization probe. The probe recognizes a single 10.0-kb band in the wild-type strain and a single 6.4-kb band in the $\triangle x \operatorname{trG}$ strain. Diagram (C.) and Southern blot (D.) of the wild-type and $\Delta x t r H$ strains when digested with ECoRI. A 1-kb DNA fragment from the xtrH 5'UTR (untranslated) region was used as a hybridization probe. The probe recognizes a single 3.4-kb band in the wild-type strain and a single $3.0-\mathrm{kb}$ band in the $\triangle \mathrm{xtrH}$ strain. Diagram (E.) and Southern blot (F.) of the wild-type and $\triangle c l t B$ strains when digested with Xbal. A 1-kb DNA fragment from the cltB $5^{\prime} \cup T R$ (untranslated) region was used as a hybridization probe. The probe recognizes a single 2.0-kb band in the wild-type strain and a single 3.3-kb band in the $\triangle c l t B$ strain. Diagram (G.) and Southern blot $(H$.) of the wild-type and $\triangle c l t A$ $\triangle c l t B$ strains when digested with $K p n l$. A 1-kb DNA fragment from the $c l t B$ $3^{\prime} U T R$ (untranslated) region was used as a hybridization probe. The probe recognizes a single $2.0-\mathrm{kb}$ band in the wild-type strain and a single $2.5-\mathrm{kb}$ band in the $\triangle x \operatorname{tr} G$ strain.

Additional file 6. PCR confirmation of the $\triangle c l t B: .: C l t B^{+}$strain. Genomic DNA from $A$. nidulans wild-type, complementing strains $\triangle \mathrm{clt} B:: \mathrm{Cl} B^{+}$ (candidates 1, 2, and 3 ) and deletion strain $\triangle c l t B$ were isolated and used as template for PCR reactions. (A) Specific primers P70 and P31 were used to amplify a DNA fragment of about $3.1 \mathrm{~kb}$. (B) Specific primers P70 and P37 were used to amplify a DNA fragment of about $3.0 \mathrm{~kb}$. Lanes 1 and 7: Cnegative control with no DNA as a template; lanes 2 and 8: genomic DNA from the $\triangle C l t B: .: C l t B$ candidate 1; lanes 3 and 9: genomic DNA from the $\triangle c l t B: .: \mathrm{lt} B$ candidate 2 ; lanes 4 and 10: genomic DNA from the $\triangle \mathrm{clt} B .: \mathrm{clt} B$ candidate 3 ; lanes 5 and 11: genomic DNA from the $\triangle$ cltB deletion strain; lanes 6 and 12: genomic DNA from the wild-type strain. The * indicates the candidate containing the homologous integration of the complementing cassette and $\left(\mathrm{C}^{-}\right)$the negative control. 


\section{Abbreviations}

2G: second-generation bioethanol; BP: base pairs; cDNA: complementary DNA; CDT: cellodextrin transporter; CLT: cellobiose transporter; CMC: carboxymethylcellulose; EDTA: ethylenediaminetetraacetic acid; GFP: green fluorescent protein; HXT: hexose transporter; Kb: kilo bases; $K_{m}$ : Michaelis constant; M: molar; MFS: major facilitator superfamily; MM: minimal medium; Nm: nanometre; OD: optical densisty; ORF: open reading frame; PBS: phosphatebuffered saline; PCR: polymerase chain reaction; pyrG: orotidine-5'-phosphate decarboxylase gene; Pyro: pyridoxine gene; RT-qPCR: quantitative reverse transcription PCR; TRP: tryptophan; $\mathrm{V}_{\max }$ : maximum reaction velocity rate; $X D H$ :

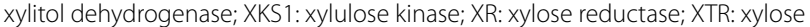
transporter; YNB: yeast nitrogen base.

\section{Authors' contributions}

GHG, TFR, NSP, PBAL, FBM, and JVCO contributed to design, acquisition and analysis of data. LNAR, TFR, and GHG contributed to the concept and design of the investigation in addition to the preparation of the manuscript. All authors read and approved the final manuscript.

\section{Author details}

${ }^{1}$ Departamento de Ciências Farmacêuticas, Faculdade de Ciências Farmacêuticas de Ribeirão Preto, Universidade de São Paulo, Av. do Café S/N, Ribeirão Preto, SP CEP 14040-903, Brazil. ${ }^{2}$ Departamento de Biologia Celular, Instituto de Biologia, Universidade de Brasília, Brasília CEP 70910-900, Brazil. ${ }^{3}$ Laboratório Nacional de Ciência e Tecnologia do Bioetanol (CTBE), Campinas, Brazil.

\section{Acknowledgements}

We would like to thank Dr. Eckardt Boles for providing the EBY.VW4000 yeast strain, Dr. Jamie Cate for providing the plasmids containing the N. crassa $\beta$-glucosidase and CDT-1 genes, Dr. Ana Cristina Colabardini and Santiago Latar for providing the initial material to carry out part of the experiments in this study. We also would like to thank the three anonymous reviewers for their comments and suggestions.

\section{Competing interests}

The authors declare that they have no competing interests.

\section{Funding}

We would like to thank the Conselho Nacional de Desenvolvimento Científico e Tecnológico (CNPq) and the Fundação de Amparo à Pesquisa do Estado de São Paulo (FAPESP) in Brazil for funding this research.

Received: 18 December 2015 Accepted: 6 September 2016 Published online: 26 September 2016

\section{References}

1. Zaldivar J, Nielsen J, Olsson L. Fuel ethanol production from lignocellulose: a challenge for metabolic engineering and process integration. Appl Microbiol Biotechnol. 2001;56:17-34.

2. Ohara H. Biorefinery. Appl Microbiol Biotechnol. 2003;62:474-7.

3. Ragauskas AJ, Williams CK, Davison BH, Britovsek G, Cairney J, Eckert CA, Frederick WJ, Hallett JP, Leak DJ, Liotta CL, Mielenz JR, Murphy R, Templer $\mathrm{R}$, Tschaplinski T. The path forward for biofuels and biomaterials. Science. 2006;311:484-9.

4. Alvira P, Tomás-Pejó E, Ballesteros M, Negro MJ. Pretreatment technologies for an efficient bioethanol production process based on enzymatic hydrolysis: a review. Bioresour Technol. 2010;101:4851-61.

5. Dashtban M, Schraft H, Qin W. Fungal bioconversion of lignocellulosic residues; opportunities and perspectives. Int J Biol Sci. 2009:5:578-95.

6. Jordan DB, Bowman MJ, Braker JD, Dien BS, Hector RE, Lee CC, Mertens JA, Wagschal K. Plant cell walls to ethanol. Biochem J. 2012;442:241-52.

7. Hahn-Hägerdal B, Galbe M, Gorwa-Grauslund MF, Lidén G, Zacchi G. Bio-ethanol - the fuel of tomorrow from the residues of today. Trends Biotechnol. 2006:24:549-56.

8. Liu Y-S, Baker JO, Zeng Y, Himmel ME, Haas T, Ding S-Y. Cellobiohydrolase hydrolyzes crystalline cellulose on hydrophobic faces. J Biol Chem. 2011;286:11195-201.
9. Mathew GM, Sukumaran RK, Singhania RR, Pandey A. Progress in research on fungal cellulases for lignocellulose degradation. J Sci Ind Res. 2008;67:898-907.

10. Nevoigt E. Progress in metabolic engineering of Saccharomyces cerevisiae. Microbiol Mol Biol Rev. 2008:72:379-412.

11. Young E, Lee S-M, Alper H. Optimizing pentose utilization in yeast: the need for novel tools and approaches. Biotechnol Biofuels. 2010:3:24

12. Galazka JM, Tian C, Beeson WT, Martinez B, Glass NL, Cate JHD. Cellodextrin transport in yeast for improved biofuel production. Science. 2010;330:84-6.

13. Young EM, Comer AD, Huang H, Alper HS. A molecular transporter engineering approach to improving xylose catabolism in Saccharomyces cerevisiae. Metab Eng. 2012;14:401-11.

14. Nieves LM, Panyon LA, Wang X. Engineering sugar utilization and microbial tolerance toward lignocellulose conversion. Front Bioeng Biotechnol. 2015:3:1-10.

15. Carroll A, Somerville C. Cellulosic biofuels. Annu Rev Plant Biol. 2009;60:165-82.

16. Pao SS, Paulsen IT, Saier MH. Major facilitator superfamily. Microbiol Mol Biol Rev. 1998:62:1-34.

17. Yan N. Structural advances for the major facilitator superfamily (MFS) transporters. Trends Biochem Sci. 2013:38:151-9.

18. Gárdonyi M, Jeppsson M, Lidén G, Gorwa-Grauslund MF, Hahn-Hägerdal B. Control of xylose consumption by xylose transport in recombinant Saccharomyces cerevisiae. Biotechnol Bioeng. 2003;82:818-24.

19. Kim SR, Ha SJ, Wei N, Oh EJ, Jin YS. Simultaneous co-fermentation of mixed sugars: a promising strategy for producing cellulosic ethanol. Trends Biotechnol. 2012;30:274-82.

20. Nijland JG, Shin HY, de Jong RM, de Waal PP, Klaassen P, Driessen AJ. Engineering of an endogenous hexose transporter into a specific D-xylose transporter facilitates glucose-xylose co-consumption in Saccharomyces cerevisiae. Biotechnol Biofuels. 2014;7:168.

21. Farwick A, Bruder S, Schadeweg V, Oreb M, Boles E. Engineering of yeast hexose transporters to transport D-xylose without inhibition by D-glucose. Proc Natl Acad Sci. 2014;111:5159-64.

22. Shin HY, Nijland JG, de Waal PP, de Jong RM, Klaassen P, Driessen AJM. An engineered cryptic Hxt11 sugar transporter facilitates glucose-xylose co-consumption in Saccharomyces cerevisiae. Biotechnol Biofuels. 2015;8:176

23. Young EM, Comer AD, Huang H, Alper HS. A molecular transporter engineering approach to improving xylose catabolism in Saccharomyces cerevisiae. Metab Eng. 2012;14:401-11.

24. Hector RE, Qureshi N, Hughes SR, Cotta M. Expression of a heterologous xylose transporter in a Saccharomyces cerevisiae strain engineered to utilize xylose improves aerobic xylose consumption. Appl Microbiol Biotechnol. 2008:80:675-84.

25. Du J, Li S, Zhao H. Discovery and characterization of novel D-xylose-specific transporters from Neurospora crassa and Pichia stipitis. Mol BioSyst. 2010;6:2081-340.

26. Galazka JM, Tian C, Beeson WT, Martinez B, Glass NL, Cate JH. Cellodextrin transport in yeast for improved biofuel production. Science. 2010;330:84-6.

27. Kim H, Lee W-H, Galazka JM, Cate JHD, Jin Y-S. Analysis of cellodextrin transporters from Neurospora crassa in Saccharomyces cerevisiae for cellobiose fermentation. Appl Microbiol Biotechnol. 2014;98:1087-94.

28. van den Brink J, de Vries RP. Fungal enzyme sets for plant polysaccharide degradation. Appl Microbiol Biotechnol. 2011;91:1477-92.

29. Culleton H, McKie V, de Vries RP. Physiological and molecular aspects of degradation of plant polysaccharides by fungi: what have we learned from Aspergillus? Biotechnol J. 2013;8:884-94.

30. Ries L, Pullan ST, Delmas S, Malla S, Blythe MJ, Archer DB. Genome-wide transcriptional response of Trichoderma reesei to lignocellulose using RNA sequencing and comparison with Aspergillus niger. BMC Genom. 2013;14:541.

31. Ferreira ME, Colombo AL, Paulsen I, Ren Q, Wortman J, Huang J, Goldman $\mathrm{MH}$, Goldman $\mathrm{GH}$. The ergosterol biosynthesis pathway, transporter genes, and azole resistance in Aspergillus fumigatus. Med Mycol. 2005:43(Suppl 1):S313-9.

32. Zhang W, Kou Y, Xu J, Cao Y, Zhao G, Shao J, Wang H, Wang Z, Bao X, Chen G, Liu W. Two major facilitator superfamily sugar transporters from 
Trichoderma reesei and their roles in induction of cellulase biosynthesis. J Biol Chem. 2013;288:32861-72.

33. Colabardini A, Ries LN, Brown N, dos Reis T, Savoldi M, Goldman MHS, Menino J, Rodrigues F, Goldman G. Functional characterization of a xylose transporter in Aspergillus nidulans. Biotechnol Biofuels. 2014;7:46.

34. Nehls U, Wiese J, Guttenberger M, Hampp R. Carbon allocation in ectomycorrhizas: identification and expression analysis of an Amanita muscaria monosaccharide transporter. Mol Plant Microbe Interact. 1998;11:167-76.

35. Voegele RT, Struck C, Hahn M, Mendgen K. The role of haustoria in sugar supply during infection of broad bean by the rust fungus Uromyces fabae. Proc Natl Acad Sci USA. 2001;98:8133-8.

36. Delgado-Jarana J, Moreno-Mateos MA, Benítez T. Glucose uptake in Trichoderma harzianum: role of gtt1. Eukaryot Cell. 2003;2:708-17.

37. Xie X, Wilkinson HH, Correa A, Lewis Z, Bell-Pedersen D, Ebbole DJ. Transcriptional response to glucose starvation and functional analysis of a glucose transporter of Neurospora crassa. Fungal Genet Biol. 2004:41:1104-19.

38. Madi L, McBride SA, Bailey LA, Ebbole DJ. rco-3, a gene involved in glucose transport and conidiation in Neuropsora crassa. Genetics. 1997;146:499-508.

39. Vankuyk PA, Diderich JA, MacCabe AP, Hererro O, Ruijter GJG, Visser J. Aspergillus niger mstA encodes a high-affinity sugar/H+ symporter which is regulated in response to extracellular pH. Biochem J. 2004;379(2):375-83.

40. Wei H, Vienken $K$, Weber R, Bunting S, Requena N, Fischer R. A putative high affinity hexose transporter, hxtA, of Aspergillus nidulans is induced in vegetative hyphae upon starvation and in ascogenous hyphae during cleistothecium formation. Fungal Genet Biol. 2004;41:148-56.

41. Kim SR, Ha S-J, Wei N, Oh EJ, Jin Y-S. Simultaneous co-fermentation of mixed sugars: a promising strategy for producing cellulosic ethanol. Trends Biotechnol. 2012;30:274-82.

42. Znameroski EA, Li X, Tsai JC, Galazka JM, Glass NL, Cate JHD. Evidence for transceptor function of cellodextrin transporters in Neurospora crassa.J Biol Chem. 2014;289:2610-9.

43. Fang F, Salmon K, Shen MW, Aeling KA, Ito E, Irwin B, Tran UP, Hatfield GW, Da Silva NA, Sandmeyer S. A vector set for systematic metabolic engineering in Saccharomyces cerevisiae. Yeast. 2011;28:123-36.

44. Shen MW, Fang F, Sandmeyer S, Da Silva NA. Development and characterization of a vector set with regulated promoters for systematic metabolic engineering in Saccharomyces cerevisiae. Yeast. 2012;29:495-503.

45. Hong SJ, Kim HJ, Kim JW, Lee DH, Seo JH. Optimizing promoters and secretory signal sequences for producing ethanol from inulin by recombinant Saccharomyces cerevisiae carrying Kluyveromyces marxianus inulinase. Bioprocess Biosyst Eng. 2015;38:263-72.

46. dos Reis TF, Menino JF, Bom VLP, Brown NA, Colabardini AC, Savoldi M, Goldman MHS, Rodrigues F, Goldman GH. Identification of glucose transporters in Aspergillus nidulans. PLOS ONE. 2013;8:1-15.

47. Vankuyk PA, Diderich JA, MacCabe AP, Hererro O, Ruijter GJ, Visser J. Aspergillus niger mstA encodes a high-affinity sugar/H+ symporter which is regulated in response to extracellular pH. Biochem J. 2004;379:375-83.

48. Wieczorke R, Krampe S, Weierstall T, Freidel K, Hollenberg CP, Boles E. Concurrent knock-out of at least 20 transporter genes is required to block uptake of hexoses in Saccharomyces cerevisiae. FEBS Lett. 1999;464:123-8.

49. Kubicek CP, Messner R, Gruber F, Mach RL, Kubicek-Pranz EM. The Trichoderma cellulase regulatory puzzle: from the interior life of a secretory fungus. Enzym. 1993;15:90-9.

50. Vaheri $M$, Vaheri M, Kauppinen V. Formation and release of cellulolytic enzymes during growth of Trichoderma reesei on cellobiose and glycerol. Eur J Appl Microbiol Biotechnol. 1979;8:73-80.

51. Znameroski EA, Coradetti ST, Roche CM, Tsai JC, lavarone AT, Cate JHD, Glass NL. Induction of lignocellulose-degrading enzymes in Neurospora crassa by cellodextrins. Proc Natl Acad Sci. 2012;109:6012-7.
52. Zhou Q, Xu J, Kou Y, Lv X, Zhang X, Zhao G, Zhang W, Chen G, Liu W. Differential involvement of $\beta$-glucosidases from Hypocrea jecorina in rapid induction of cellulase genes by cellulose and cellobiose. Eukaryot Cell. 2012;1:1371-81.

53. Li M, Tu M, Cao D, Bass P, Adhikari S. Distinct roles of residual xylan and lignin in limiting enzymatic hydrolysis of organosolv pretreated loblolly pine and sweetgum. J Agric Food Chem. 2013;61:646-54.

54. Li X, Yu VY, Lin Y, Chomvong K, Estrela R, Park A, Liang JM, Znameroski EA, Feehan J, Kim SR, Jin YS, Glass NL, Cate JH. Expanding xylose metabolism in yeast for plant cell wall conversion to biofuels. Elife. 2015;4:5896.

55. Gielkens MM, Dekkers E, Visser J, de Graaff LH. Two cellobiohydrolaseencoding genes from Aspergillus niger require $\mathrm{D}$-xylose and the xylanolytic transcriptional activator XInR for their expression. Appl Environ Microbiol. 1999;65:4340-5.

56. Forment JV, Flipphi M, Ventura L, González R, Ramón D, Maccabe AP. High-affinity glucose transport in Aspergillus nidulans is mediated by the products of two related but differentially expressed genes. PLOS ONE. 2014;9:e94662.

57. Forment JV, Flipphi M, Ramón D, Ventura L, Maccabe AP. Identification of the $m s t E$ gene encoding a glucose-inducible, low affinity glucose transporter in Aspergillus nidulans. J Biol Chem. 2006;281:8339-46.

58. Young EM, Tong A, Bui H, Spofford C, Alper HS. Rewiring yeast sugar transporter preference through modifying a conserved protein motif. Proc Natl Acad Sci USA. 2014;111:131-6.

59. Ha S-J, Wei Q, Kim SR, Galazka JM, Cate J, Jin Y-S. Cofermentation of cellobiose and galactose by an engineered Saccharomyces cerevisiae strain. Appl Environ Microbiol. 2011;77:5822-5.

60. Ha S, Galazka JM, Kim SR, Choi J, Yang X, Seo J, Glass NL, Cate JHD, Jin Y-S. Engineered Saccharomyces cerevisiae capable of simultaneous cellobiose and xylose fermentation. Proc Natl Acad Sci USA. 2011;108:504-9.

61. Sambrook J, Russell DW. Molecular cloning: a laboratory manual. Cold Spring Harbor: Cold Spring Harbor Laboratory Press; 2001.

62. Colot HV, Park G, Turner GE, Ringelberg C, Crew CM, Litvinkova L, Weiss RL, Borkovich KA, Dunlap JC. A high-throughput gene knockout procedure for Neurospora reveals functions for multiple transcription factors. Proc Natl Acad Sci USA. 2006;103:10352-7.

63. Schiestl RH, Gietz RD. High efficiency transformation of intact yeast cells using single stranded nucleic acids as a carrier. Curr Genet. 1989;16:339-46.

64. Osmani SA, May GS, Morris R. Aspergillus nidulans. J Cell Biol. 1987;104:1495-504.

65. Teepe AG, Loprete DM, He Z, Hoggard TA, Hill TW. The protein kinase C orthologue PkcA plays a role in cell wall integrity and polarized growth in Aspergillus nidulans. Fungal Genet Biol. 2007:44:554-62.

66. Hector RE, Dien BS, Cotta MA, Qureshi N. Engineering industrial Saccharomyces cerevisiae strains for xylose fermentation and comparison for switchgrass conversion. J Ind Microb Biotechnol. 2011;38:1193-202.

67. Goldman GH, Morris R. Aspergillus nidulans as a model system for cell and molecular biology studies. Methods in Molecular Genetics. 1995;6:48-65.

68. Semighini CP, Marins M, Goldman MHS, Goldman GH. Quantitative analysis of the relative transcript levels of $A B C$ transporter Atr genes in Aspergillus nidulans by real-time reverse transcription-PCR assay. Appl Environ Microbiol. 2002;68:1351-7.

69. Bernt E, Gutmann I. Methods of enzymatic analysis. New York: Academic press; 1974

70. Nayak T. A versatile and efficient gene-targeting system for Aspergillus nidulans. Genetics. 2005;172:1557-66.

71. Chaveroche MK, Ghigo JM, d'Enfert C. A rapid method for efficient gene replacement in the filamentous fungus Aspergillus nidulans. Nucleic Acids Res. 2000;28:E97. 